\title{
BIAXIAL PHASES OF BENT-CORE LIQUID CRYSTAL POLYMERS IN SHEAR FLOWS*
}

\author{
SARTHOK SIRCAR ${ }^{\dagger}$, JUN LI ${ }^{\ddagger}$, AND QI WANG $\$$
}

\begin{abstract}
We develop a kinetic theory of the biaxial phases for the bent-core or V-shaped liquid crystal polymer solution (VLCPS). The Brownian motion of the biaxial molecules, biaxial molecular excluded interaction, and the rotary convection are modeled explicitly. Using the hydrodynamic theory, we study the flow-driven orientational dynamics and the corresponding rheological response in selected regimes of plane shear flows, strength of intermolecular interaction, and the bent-angle of the molecule. We identify three steady state biaxial phases and three periodic motions at various regimes of the shear rate and strength of the intermolecular potential. Phase transition sequences among the orientational phases and motions are documented with respect to the strength of the intermolecular potential at fixed bent angles. The effect of the molecular configuration (or the bentangle) of the $\mathrm{V}$-shaped molecule on mesoscopic phases and their transition sequences is investigated as well.
\end{abstract}

Key words. Kinetic theory, biaxiality, V-shaped/bent-core liquid crystal polymers, shear flows, orientational dynamics, steady states, periodic motions, rheology.

AMS subject classifications. $82 \mathrm{D} 30,76 \mathrm{M} 20$

\section{Introduction}

The phase of matter is determined by its fundamental constituents, the molecules, and their geometric configuration and mutual interactions. In the 1970s, biaxial nematogens in liquid crystals was theoretically predicted to exhibit mesoscopic biaxiality at equilibrium under certain conditions besides the uniaxial symmetry reported earlier $[26,37,21]$. The theoretical prediction of the biaxial phase of biaxial nematogens by Freiser [21] was confirmed by computer simulations of biaxial liquid crystals in the 1990 s and the early 21 st century $[2,6,10,5,30]$. It was not until 2004, when several groups of experimentalists independently discovered/confirmed the biaxial phase using different biaxial nematogens $[27,1,28,32,16]$. In addition to the thermotropic biaxial liquid crystals discovered recently $[27,1,28,32,16]$, there are also lyotropic biaxial liquid crystal systems exhibiting biaxial phases being identified in the past as well $[42,4,17]$.

In the past, research on biaxial liquid crystals and their interactions with external fields was primarily focused either on uniaxial phases of biaxial molecules [39] or on flow or external field induced biaxial phases of purely uniaxial molecules $[20,41,18,19]$. Very few studies addressed the biaxial phases due to the molecular biaxiality. Leslie and his coworkers [24, 9] extended the well-known Ericksen-Leslie continuum theory to biaxial liquid crystals, where they derived the theory based on a single second order tensor. Their focus was to extend the distortional Oseen-Frank elasticity to inhomogeneous biaxial liquid crystals to study their macroscopic morphology due to the long-range distortional elasticity while neglecting the inherent molecular elasticity. Straley studied the uniaxial and biaxial phase of biaxial nematogens using a mean-field theory focusing on the phase behavior of biaxial liquid crystals exclusively due to the molecular biaxiality and elasticity [37]. Recently, Virga

\footnotetext{
*Received: Feb 13, 2009; Accepted (in revised version): May 6, 2009.

${ }^{\dagger}$ Department of Mathematics, University of Utah, Salt Lake City, UT 84112 ,

${ }^{\ddagger}$ School of Mathematics, Nankai University, Tianjin, P.R. China, 300071.

$\S$ Department of Mathematics and NanoCenter at USC, University of South Carolina, Columbia, SC 29208, (qwang@math.sc.edu). Corresponding author.
} 
et al. [36, 11, 7, 23] developed a self-consistent mean field theory for thermotropic biaxial liquid crystals employing two second order, trace-less biaxial tensors that account for both the intrinsic biaxiality and the induced mesoscopic phase biaxiality at equilibrium. The focus of the studies was on the phases both uniaxial and biaxial exclusively due to the short-range molecular interaction or molecular elasticity. These models demonstrate that either a probability density function for three Euler angles or more than one second order tensor order parameters are necessary to fully describe the rotational orientation of an ensemble of rigid molecules of biaxiality at the mesoscale.

For bent-core biaxial liquid crystal systems, more new mesoscopic phases are yet to be experimentally found. However, they have shown some extraordinary promises in their applications in ultrafast switch technology and new display devices in the already discovered biaxial liquid crystal materials. Some of this applications involve processing of the material in flowing conditions. It is therefore important to develop hydrodynamic theories to understand the material system and genesis for morphology development in inhomogeneous biaxial liquid crystal flows and the corresponding rheological consequences. Recently, we extended the work of Straley and Virga et al on thermodynamic mean-field theories $[37,36]$ to allow the hydrodynamic coupling between the biaxial nematogens and small molecule solvent in solutions of ellipsoidal or brick-shaped biaxial liquid crystal polymers to study shear induced phases, motions, and phase transitions as well as associated rheological responses of the sheared nematic biaxial liquid crystal polymer system [33, 34]. This paper aims at deriving an analogous hydrodynamic model to address the issue on how biaxiality at the molecular scale, molecular asymmetry (the bent-core configuration), and their interaction with small molecule solvent can impact the mesoscopic phase and rheology in sheared monodomain bent-core biaxial liquid crystal polymers. We model the biaxial molecule as a rigid $\mathrm{V}$-shaped body immersed in small molecule solvent. Using a kinetic theory approach, we derive the transport equation for the orientational probability density (or distribution) function for the biaxial liquid crystal polymer, known as the Smoluchowski equation, and the stress constitutive equation to allow a full coupling with the macroscopic momentum and mass transport. The current model refines our previous hydrodynamic model for biaxial liquid crystal polymers and provides detailed kinetic calculations of the phases and rheological responses in shear flows at various model parameters as well as the varying bent-angle in the molecule $[8,33,34]$.

The paper is organized as follows. In section 2, we describe the kinetic theory for flows of bent-core biaxial liquid crystal polymers and, especially, present the Smoluchowski equation for homogenous flow of biaxial liquid crystal polymers, modeled as V-shaped suspensions in viscous solvent (section 2.1), and derive the associated stress constitutive equation (section 2.2). In section 3, we describe the Wigner-Galerkin spectral method developed to solve the Smoluchowski equation numerically. In section 4, we report and discuss various aspects of the numerical results in sheared bent-core biaxial liquid crystal polymers: the presence of the newly discovered timeperiodic motions in addition to the steady-states; the associated nematic order and the mixed moments or correlation matrices with respect to the orientational distribution function, which are used to describe the mesoscale material structures in fast motion ESR [29] and NMR [14] experiments . The rheological response of this sheared liquid crystal system is elucidated therein as well. 


\section{Kinetic theory for V-shaped LCPs}

In this section, we derive the kinetic theory for the monodomain solution of the $\mathrm{V}$-shaped liquid crystal polymer in biaxial phases. The elastic stress expression is derived using a virtual work principle while the viscous stress is calculated using an energy dissipation method. The elastic and viscous constitutive stress equation couples the kinetic equation to the transport of the macroscopic mass and momentum equation to yield the governing system of equations for the VLCP system.

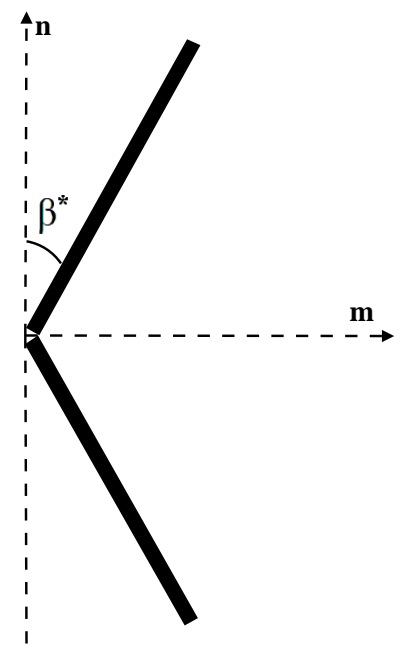

FIG. 2.1. A schematic $V$-shaped molecule with bent angle $\left(\beta^{*}\right)$. The unit vectors $(\mathbf{m}, \mathbf{n})$ denote the molecular axes. $\mathbf{n}$ is known as the direction of the bow and $\mathbf{m}$ points to the opposite direction to the arrow.

2.1. Smoluchowski equation. We model the V-shaped LCP molecule by two rigid massless arms of length a and $\mathrm{b}$, respectively, with a bent angle $\beta^{*}$ shown in figure 2.1. $\mathbf{m}$ and $\mathbf{n}$ are two mutually orthogonal unit vectors in the plane spanned by the arms of the biaxial molecule as shown in figure 2.1. When $a=b, \mathbf{m}$ is a unit vector in the opposite direction of the arrow and $\mathbf{n}$ is a unit vector in the direction of the bow. In this theory, we assume $a=b$. However, we present some results in the more general case where $\mathrm{a}$ is not necessarily equal to $\mathrm{b}$ in the derivation below. $\mathbf{k}=\mathbf{m} \times \mathbf{n}$ is the third unit vector perpendicular to both $\mathbf{m}$ and $\mathbf{n}$. The orientation of the rigid molecule is completely determined by the triad of unit vectors $(\mathbf{m}, \mathbf{n}, \mathbf{k})$. In a fixed Cartesian frame $(x, y, z)$, the three unit vectors can be parametrized by three Euler angles $\Omega=(\alpha, \beta, \gamma)$ :

$$
\begin{aligned}
& \mathbf{m}=(\cos \alpha \sin \beta, \sin \alpha \sin \beta, \cos \beta)^{T}, \\
& \mathbf{n}=(\cos \alpha \cos \beta \cos \gamma-\sin \alpha \sin \gamma, \sin \alpha \cos \beta \cos \gamma+\cos \alpha \sin \gamma,-\sin \beta \cos \gamma)^{T}, \\
& \mathbf{k}=(-\cos \alpha \cos \beta \sin \gamma-\sin \alpha \cos \gamma,-\sin \alpha \cos \beta \sin \gamma+\cos \alpha \cos \gamma, \sin \beta \sin \gamma)^{T},
\end{aligned}
$$

where $T$ denotes the transpose of a matrix or a vector.

Let $f(\Omega, t)$ be the orientational probability density function (PDF) of the Vshaped, rigid polymer nematogens (or molecules). The transport of the PDF is 
governed by the Smoluchowski equation in the mesoscale accounting for the configurational space flux due to the Brownian rotation of the molecules, the excluded volume interaction, and the flow-induced convection. The Smoluchowski equation in the molecular (or rotating) frame $(\mathbf{m}, \mathbf{n}, \mathbf{k})$ is given by:

$$
\frac{\partial}{\partial t} f+\nabla(\mathbf{v} f)=\mathbf{L}^{*} D_{r} \cdot\left(\mathbf{L} f+\frac{1}{k_{B} T} f \mathbf{L} \mathcal{U}\right)-\mathbf{L}^{*} \cdot(\mathbf{g} f)
$$

where

$$
D_{r}=D_{r 0} \operatorname{diag}\left(\frac{1}{2 \cos ^{2} \beta^{*}}, \frac{3}{2 \sin ^{2} \beta^{*}}, \frac{3}{2\left(3-2 \sin ^{2} \beta^{*}\right)}\right)
$$

is the rotational diffusivity (assumed a constant diagonal matrix), $\frac{3 D_{r 0}}{2 \sin ^{2} \beta^{*}}$ is the rotational diffusivity of a rigid rod of length $2 \mathrm{a}, a=b$ is assumed, $k_{B}$ is the Boltzmann constant, $T$ is the absolute temperature, $\mathbf{g}$ is the complex angular velocity due to the flow of velocity $\mathbf{v}$ given by

$\mathbf{g}=i\left[\mathbf{m}(\nabla \mathbf{v}: \mathbf{n k})-\mathbf{n}(\nabla \mathbf{v}: \mathbf{m k})+\frac{\mathbf{k}}{1+2 \cos ^{2} \beta^{*}}\left(\sin ^{2} \beta^{*} \nabla \mathbf{v}: \mathbf{m n}-3 \cos ^{2} \beta^{*} \nabla \mathbf{v}: \mathbf{n m}\right)\right]$,

where the contraction operator is defined in this paper by $\mathbf{A}: \mathbf{B}=\operatorname{tr}\left(\mathbf{A} \cdot \mathbf{B}^{T}\right), \nabla \mathbf{v}$ is the velocity gradient, and $\mathbf{L}=i \mathbf{x} \times \frac{\partial}{\partial \mathbf{x}}$ is the angular momentum operator whose components in the rotating frame are

$$
\begin{aligned}
& L_{m}=i \frac{\partial}{\partial \gamma}, \\
& L_{n}=i\left(\cos \gamma \cot \beta \frac{\partial}{\partial \gamma}+\sin \gamma \frac{\partial}{\partial \beta}-\frac{\cos \gamma}{\sin \beta} \frac{\partial}{\partial \alpha}\right), \\
& L_{k}=i\left(\sin \gamma \cot \beta \frac{\partial}{\partial \gamma}+\cos \gamma \frac{\partial}{\partial \beta}+\frac{\sin \gamma}{\sin \beta} \frac{\partial}{\partial \alpha}\right) .
\end{aligned}
$$

$\mathcal{U}$ is the "biaxial" excluded volume or steric interaction potential given by the generalized quadratic potential (a linear variant of the straley's pair-potential depending on the bent angle $\beta^{*}[37]$ )

$$
\mathcal{U}=-\frac{3}{2} N k_{B} T\left[\xi_{0} \mathbf{M}: \mathbf{m m}+\gamma_{0}(\mathbf{N}: \mathbf{m m}+\mathbf{M}: \mathbf{n n})+\lambda_{0} \mathbf{N}: \mathbf{n n}\right]
$$

where $\mathbf{M}=\langle\mathbf{m m}\rangle$ and $\mathbf{N}=\langle\mathbf{n n}\rangle$ are the second moment tensors of $\mathbf{m}$ and $\mathbf{n}$ with respect to the pdf respectively, the symbol

$$
\langle(\bullet)\rangle=\int(\bullet) f(\Omega) d \Omega
$$

denotes the ensemble average with respect to the $\operatorname{pdf} f$, and $N$ is a dimensionless parameter measuring the strength of the intermolecular potential and proportional to the concentration of VLCP molecules. The material parameters $\left(\xi_{0}, \gamma_{0}, \lambda_{0}\right)$ are linearly related to the parameters $(\gamma, \lambda)$ of the Straley's pair-potential:

$$
\xi_{0}=1+2 \gamma+\lambda, \gamma_{0}=2(\gamma+\lambda), \lambda_{0}=4 \lambda .
$$

In the V-shaped or bent-core liquid crystal theory, however, these material parameters are functions of the bent-angle $\left(\beta^{*}\right)$ according to Teixeira et al. [38]. We briefly 
outline these relations (the details of the expression for the material parameters $(\gamma, \lambda)$ are derived in [38]):

$$
\begin{aligned}
& \xi_{0}=\frac{11}{4}+\left|\cos \beta^{*}\right|+\frac{3}{4}\left|\cos 2 \beta^{*}\right|-\frac{1}{2}\left|\sin \left(\cos ^{-1}\left(\sin ^{2} \beta^{*}\right)\right)\right|-3\left|\sin \left(\cos ^{-1}\left(\frac{1}{2} \sin 2 \beta^{*}\right)\right)\right| \\
& \gamma_{0}=\frac{5}{2}+\left|\cos \beta^{*}\right|+\frac{3}{2}\left|\cos 2 \beta^{*}\right|+\left|\sin \left(\cos ^{-1}\left(\sin ^{2} \beta^{*}\right)\right)\right|-6\left|\sin \left(\cos ^{-1}\left(\frac{1}{2} \sin 2 \beta^{*}\right)\right)\right| \\
& \lambda_{0}=3\left(1+\left|\cos 2 \beta^{*}\right|+2\left|\sin \left(\cos ^{-1}\left(\sin ^{2} \beta^{*}\right)\right)\right|-4\left|\sin \left(\cos ^{-1}\left(\frac{1}{2} \sin 2 \beta^{*}\right)\right)\right|\right) .
\end{aligned}
$$

We note that the potential represents only the second order interaction since we wish to present only the biaxial phases of these V-shaped liquid crystals. Vector (the first moment) and higher order (the third moment) interactions, which are also observed in these liquid crystals [25] and correspond to a symmetry other than the biaxial $D_{2 h}$ symmetry, are neglected and will be considered in a forthcoming paper. The free energy for the ensemble of the V-shaped molecules in solutions, consisting of the rotational Brownian motion and the excluded volume interaction, is given by

$$
\mathcal{A}[f]=\nu \int_{\Omega}\left[k_{B} T(\ln f-1)+\frac{\mathcal{U}}{2}\right] f d \Omega,
$$

where $\nu$ is the number density of the VLCP molecules in the system and $\Omega$ is the domain that the VLCP solution occupies.

2.2. Constitutive stresses. The extra elastic stress tensor for the VLCP system is calculated by an extended virtual work principle $[12,40,34]$. Here, we present the derived results only. Details can be found in [40,34]. We denote the flow vector ( $\mathrm{g}$ ) by

$$
\mathbf{g}=K: \alpha_{1} \mathbf{m}+K: \alpha_{2} \mathbf{n}+K: \alpha_{3} \mathbf{k}
$$

The elastic stress tensor can then be expressed in terms of the angular momentum operator $(\mathbf{L})$ as follows:

$$
\begin{aligned}
\tau_{e}^{\alpha \beta} & =\nu\left\langle\alpha_{1}^{* \alpha \beta} L_{m} \mu+\alpha_{2}^{* \alpha \beta} L_{n} \mu+\alpha_{3}^{* \alpha \beta} L_{k} \mu\right\rangle \\
& =\nu\left[-k_{B} T\left\langle\mathbf{L}^{*} \cdot \vec{\alpha}^{\alpha \beta}\right\rangle+\left\langle\alpha_{1}^{* \alpha \beta} L_{m} \mathcal{U}+\alpha_{2}^{* \alpha \beta} L_{n} \mathcal{U}+\alpha_{3}^{* \alpha \beta} L_{k} \mathcal{U}\right\rangle\right],
\end{aligned}
$$

where $\mu=\frac{\delta \mathcal{A}}{\nu \delta f}$ is the chemical potential of the system, $\vec{\alpha}^{\alpha \beta}=\left(\alpha_{1}^{\alpha \beta}, \alpha_{2}^{\alpha \beta}, \alpha_{3}^{\alpha \beta}\right)$ is a third order tensor and $\mathbf{L}^{*} \cdot \vec{\alpha}^{\alpha \beta}=\sum_{i=m, n, k} L_{i}^{*} \alpha_{i}^{\alpha \beta}$. There is also an elastic external force

$$
\mathbf{F}_{e}=-\nu\langle\nabla \mu\rangle .
$$

For homogeneous flows, we note that the elastic body force vanishes.

The viscous stress for the VLCPs is given by

$$
\tau_{v}=\tau_{v}^{s}+\tau_{v}^{V L C P},
$$

where $\tau_{v}^{s}=2 \eta \mathbf{D}$ is the viscous stress tensor at the zero strain rate and $\tau_{v}^{V L C P}$ is the viscous stress due to solvent and VLCP friction, which follows a model calculation involving energy dissipation $(W)$, given by:

$$
W=\mathbf{K}: \tau_{v}^{V L C P} .
$$


In this calculation, we model the $\mathrm{V}$-shaped molecule as a geometric object consisting of two joined arms, each of which is made up of a finite number of small beads of spherical shapes [12]. We note that this V-shaped molecular model used here is slightly different from the one adopted in the derivation of the Smoluchowski equation in that the arms here are not coarse-grained into two joined dumbbells of bead-springs. We derive the stress expression for the general case but present the final result only for the case where the length of each arm is the same, i.e., $a=b$. The hydrodynamic interaction due to the presence of multiple beads is neglected. Under the velocity gradient $\mathbf{K}=\nabla \mathbf{v}$, each rod rotates about the center of mass of the rigid V-shaped body with the angular velocity $\mathbf{g}_{v}=-i \mathbf{g}$ (given by equation (2.3)). The velocity of the $n^{\text {th }}$ bead (in the $k^{\text {th }}$ arm, $\mathrm{k}=1,2$ ) relative to the fluid suspension is

$$
\mathbf{V}_{n}^{k}=\mathbf{g}_{v} \times \mathbf{u}^{k}-\mathbf{K} \cdot \mathbf{u}^{k},
$$

where $\mathbf{u}^{k}$ is the distance vector of the $n^{\text {th }}$-bead in the $k^{\text {th }}$ arm measured from the center-of- mass of the system, which is given by

$$
\mathbf{x}_{C . M}=\frac{a^{2}+b^{2}}{2(a+b)} \sin \left(\beta^{*}\right) \mathbf{m}+\frac{a-b}{2} \cos \left(\beta^{*}\right) \mathbf{n} .
$$

The directional vector along each arm is given, in the general case, by

$$
\mathbf{u}^{1,2}=\left(s-\frac{a^{2}+b^{2}}{2(a+b)}\right) \sin \left(\beta^{*}\right) \mathbf{m}+\left( \pm s-\frac{a-b}{2}\right) \cos \left(\beta^{*}\right) \mathbf{n} .
$$

Hence,

$$
\begin{aligned}
& \mathbf{V}_{n}^{1}=r_{1}[(\mathbf{K}: \mathbf{m k}) \mathbf{k}-\mathbf{K} \cdot \mathbf{m}]+r_{2}[(\mathbf{K}: \mathbf{n k}) \mathbf{k}-\mathbf{K} \cdot \mathbf{n}]+\mathbf{K}: \alpha_{3 v}\left(r_{1} \mathbf{n}-r_{2} \mathbf{m}\right), \\
& \mathbf{V}_{n}^{2}=r_{1}[(\mathbf{K}: \mathbf{m k}) \mathbf{k}-\mathbf{K} \cdot \mathbf{m}]+r_{3}[(\mathbf{K}: \mathbf{n k}) \mathbf{k}-\mathbf{K} \cdot \mathbf{n}]+\mathbf{K}: \alpha_{3 v}\left(r_{1} \mathbf{n}-r_{3} \mathbf{m}\right),
\end{aligned}
$$

where $s$ is the arclength measured from the origin in figure 1 along either arm $\mathrm{a}$ or $\mathrm{b}$, and $\alpha_{3 v}=-i \alpha_{3}$ is a real second order tensor. We denote $r_{1}=(s-$ $\left.\frac{a^{2}+b^{2}}{2(a+b)}\right) \sin \left(\beta^{*}\right), r_{2,3}=\left( \pm s-\frac{a-b}{2}\right) \cos \left(\beta^{*}\right) . \quad\left(\mathbf{K}: \alpha_{k v}\right)$ is the $k^{t h}$ component of the angular velocity vector $\mathbf{g}$. We assume that the frictional force acting on each segment is $\mathbf{F}_{n}^{k}=\zeta \mathbf{V}_{n}^{k}$, where $\zeta$ is the friction coefficient. Then, the work done by the frictional force per unit time and unit volume is:

$$
W=\nu k_{B} T \sum_{n, k}\left\langle\mathbf{F}_{n}^{k} \cdot \mathbf{V}_{n}^{k}\right\rangle=\nu k_{B} T \zeta\left[\int_{0}^{a}\left\langle\mathbf{V}_{n}^{1} \cdot \mathbf{V}_{n}^{1}\right\rangle d s+\int_{0}^{b}\left\langle\mathbf{V}_{n}^{2} \cdot \mathbf{V}_{n}^{2}\right\rangle d s\right],
$$

where the discrete sum is replaced by a continuous integration along the arms of the V-shaped rigid body.

In the following, we limit to the case where the arm length of the molecule is symmetric (i.e., $\mathrm{a}=\mathrm{b})$. Using equations (2.12), (2.16), the viscous stress in this case is given by

$$
\begin{aligned}
\tau_{v}^{V L C P}= & \nu k_{B} T \zeta \frac{a^{3}}{6} \nabla \mathbf{v}:\left[\sin ^{2} \beta^{*}\langle\mathbf{m m m m}\rangle+4 \cos ^{2} \beta^{*}\langle\mathbf{n n n n}\rangle+\frac{\sin ^{2} \beta^{*} \cos ^{2} \beta^{*}}{\left(1+2 \cos ^{2} \beta^{*}\right)^{2}}\right. \\
& \left.\left(5 \cos ^{2} \beta^{*}+4\right)\langle(\mathbf{m n}+\mathbf{n m})(\mathbf{m n}+\mathbf{n m})\rangle\right] .
\end{aligned}
$$

The total extra stress tensor is then given by

$$
\tau=\tau_{e}+\tau_{v} .
$$


It couples the Smoluchowski equation for the pdf $\mathrm{f}$ to the macroscopic transport of the momentum

$$
\rho \frac{d \mathbf{v}}{d t}=\nabla \cdot(-p \mathbf{I}+\tau)+\mathbf{F}_{e},
$$

where $\rho$ is the density of the VLCP solution and $p$ is the hydrodynamic pressure. The velocity is subject to the constraint imposed by the incompressibility of the biaxial liquid crystal system

$$
\nabla \cdot \mathbf{v}=0 .
$$

Equations (2.2), (2.3), (2.9), (2.17)-(2.20) constitute the governing system of equations for the solution of VLCPs in the kinetic theory.

\section{Numerical methods}

We next consider the flow-driven nematodynamics of the VLCP monodomain. For any linear flow, so long as it satisfies the continuity equation, a pressure can be chosen to satisfy the conservation of the linear momentum. The stress tensor in this case is a constant, so the only equation that we need to solve is the Smoluchowski equation. Once the pdf is obtained from solving the Smoluchowski equation, the stress tensor is calculated from the stress constitutive relations by ensemble averages, which is constant in the entire monodomain. We employ a Galerkin spectral method based on the Wigner-function expansion to solve the Smoluchowski equation numerically $[33,34]$.

The equations (2.2), (2.9), (2.17) are then discretized in a generalized Fourier series using the Wigner function expansion. The product of Wigner's functions (matrices) is carried out by using the Clebsh-Gordon formula [31]. The details of derivation are presented in [34]. The resulting system of equations is an ODE system for a truncated set of generalized Fourier coefficients in the expansion. We use a fourth order Runge-Kutta method to integrate the large ODE system in time and adopt a time step of $\Delta t=10^{-3}$ in our calculations.

\section{Numerical results and discussions}

A typical V-shaped molecule with bent angle $\beta^{*}=\left(\frac{\pi}{2}-\frac{\theta}{2}\right)$ is shown in figure 2.1. The Smoluchowski equation (equation (2.2)) is nondimensionalized by the elastic time scale $t_{0}=\frac{1}{D_{r 0}}$. For an imposed shear flow field $\mathbf{v}=(\dot{\gamma} y, 0,0)$ with shear rate $\dot{\gamma}$, the nondimensionalization leads to the non-dimensional shear flow parameter (Peclet number)

$$
P e=\dot{\gamma} D_{r 0} .
$$

The dimensionless equation also contains the bent-angle $\beta^{*}$ and the material parameters associated with the excluded volume potential: $N, \xi_{0}, \lambda_{0}, \gamma_{0}$, (note that only the parameter $N$ is independent, $\xi_{0}, \lambda_{0}, \gamma_{0}$ are functions of $\beta^{*}$ and their explicit relationship is given by equation (2.6)). The dimensionless time is scaled as $\tilde{t}=\frac{t}{t_{0}}$. The stress tensors are also nondimensionalized by $\nu k_{B} T$ :

$$
\begin{aligned}
\tilde{\tau}_{e}= & -\left\langle\mathbf{L}^{*} \cdot \vec{\alpha}^{\alpha \beta}\right\rangle+\frac{1}{k_{B} T}\left[\left\langle\alpha_{1}^{* \alpha \beta} L_{m} \mathcal{U}+\alpha_{2}^{* \alpha \beta} L_{n} \mathcal{U}+\alpha_{3}^{* \alpha \beta} L_{k} \mathcal{U}\right\rangle\right], \\
\tilde{\tau}_{v}= & \frac{2}{R e} \mathbf{D}+\frac{1}{R e_{V L C P}} \nabla \mathbf{v}:\left[\sin ^{2} \beta^{*}\langle\mathbf{m m m m}\rangle+4 \cos ^{2} \beta^{*}\langle\mathbf{n n n n}\rangle+\frac{\sin ^{2} \beta^{*} \cos ^{2} \beta^{*}}{\left(1+2 \cos ^{2} \beta^{*}\right)^{2}}\right. \\
& \left.\left(5 \cos ^{2} \beta^{*}+4\right)\langle(\mathbf{m n}+\mathbf{n m})(\mathbf{m n}+\mathbf{n m})\rangle\right],
\end{aligned}
$$


where an appropriate length scale is used to nondimensionalize the velocity field $\mathbf{v}=$ $(P e y, 0,0), R e=\frac{\eta}{\nu k_{B} T t_{0}}$ is the Reynolds number for the solvent and $R e_{V L C P}=$ $\frac{6}{\zeta a^{3}}$ is the Reynolds number for the VLCP. For brevity, we will drop the tilde on dimensionless quantities in the following.

Our numerical studies are carried out in the range of shear strength $0.0 \leq P e \leq$ 14.0, bent angle values $\left(\beta^{*}=5^{\circ}, 20^{\circ}, 40^{\circ}, 50^{\circ}, 70^{\circ}\right)$, and VLCP dimensionless concentrations $N=1.25,4.9,5.5,6.28,7.84$, respectively. We arrange our discussions in this section as follows. In section 4.1, we describe the various orientational phases observed in our studies of the selected regime of the shear strength and the bent-angle values. The variation in the biaxial order and rheology vs. the nematic concentration $N$ (section 4.2) and vs. the bent-angle (section 4.3) are then discussed in the next two sections. In section 4.4 , we make an attempt to address the biaxial ordering of the V-shaped liquid crystals from the perspective of the Correlation matrices. Finally, we provide a brief discussion on how the variation of the bent-angle effects the nature of the phase transition, using the "free-energy" functional (section 4.5).

The correlation matrices provide additional mesoscopic information of molecular orientation, correlation, and the phase of these bent-core liquid crystals. In addition to the autocorrelation matrices $\langle\mathbf{m m}\rangle,\langle\mathbf{n n}\rangle,\langle\mathbf{k k}\rangle=\mathbf{I}-\langle\mathbf{m m}\rangle-\langle\mathbf{n n}\rangle$, the other correlation matrices are given by the mixed moments $(\langle\mathbf{m n}\rangle,\langle\mathbf{n k}\rangle,\langle\mathbf{k m}\rangle)$. For any pair of unit vectors $\mathbf{r}_{i}$ and $\mathbf{r}_{j}$, the effective Orientational Correlation Functions (OCF) are defined as

$$
\begin{aligned}
\phi^{\mathbf{m n}}\left(\mathbf{r}_{i}, \mathbf{r}_{j}\right) & =\mathbf{r}_{i}^{T} \cdot\langle\mathbf{m n}\rangle \cdot \mathbf{r}_{j} \\
& =\left\langle\left(\mathbf{r}_{i} \cdot \mathbf{m}\right)\left(\mathbf{r}_{j} \cdot \mathbf{n}\right)\right\rangle \\
& =\left\langle\left(\cos \angle \mathbf{r}_{i} \mathbf{m}\right)\left(\cos \angle \mathbf{r}_{j} \mathbf{n}\right)\right\rangle,
\end{aligned}
$$

which is the mean value of the product of the direction cosines of the angle between the pair of vectors $\left(\mathbf{r}_{i}, \mathbf{m}\right)$ and the pair of vectors $\left(\mathbf{r}_{j}, \mathbf{n}\right)$. Hence, $\phi^{\mathbf{m n}}\left(\mathbf{r}_{i}, \mathbf{r}_{j}\right)$ gives the correlation between the projection of $\mathbf{m}$ onto the direction $\mathbf{r}_{i}$ and $\mathbf{n}$ onto the direction $\mathbf{r}_{j}$. Similarly, its Auto Correlation Function (ACF) $\left(\psi^{\mathbf{m n}}\left(\mathbf{r}_{i}\right)=\phi^{\mathbf{m m}}\left(\mathbf{r}_{i}, \mathbf{r}_{i}\right)=\mathbf{r}_{i}\right.$. $\left.\langle\mathbf{m n}\rangle \cdot \mathbf{r}_{i}\right)$ is defined as the correlation between the degree of orientation of $\mathbf{m}$ and that of $\mathbf{n}$ in direction $\mathbf{r}_{i}$. In this paper, we restrict our qualitative analysis to vectors $\left\{\mathbf{r}_{i}, \mathbf{r}_{j}\right\} \in\left\{\mathbf{m}_{1}, \mathbf{n}_{1}, \mathbf{k}_{1}\right\}$, where $\left(\mathbf{m}_{1}, \mathbf{n}_{1}, \mathbf{k}_{1}\right)$ are the major directors of the second moments $(\mathbf{M}, \mathbf{N}, \mathbf{K})$, respectively. By a major director of a second moment, say, $\mathbf{M}$, we mean the eigenvector corresponding to the largest eigenvalue of $\mathbf{M}$. Hence, by definition, the ACFs and OCFs give measures of the correlational alignment of the molecular axes $(\mathbf{m}, \mathbf{n}, \mathbf{k})$ with respect to the mesoscale major directors $\left(\mathbf{m}_{1}, \mathbf{n}_{1}, \mathbf{k}_{1}\right)$.

4.1. Orientational phases. In the VLCP system, each molecule is subject to an elastic torque due to the mean field interaction potential and convective torque due to the imposed flow field. Different mesoscopic nematic phases arise out of the balance/imbalance between the two competing torques. These orientational phases seen in the biaxial LCP system are the steady state phases: (a) Log-Rolling LR, (b) Flow-Aligning FA, and (c) Out-of-Plane steady state OS; the time periodic phases: (d) Mixed-Kayaking MK, (e) Fluttering-Kayaking FK and (f) Wagging-Kayaking WK. Our numerical investigations only capture the chaotic phase $\mathbf{C H}$ in the limit $\beta^{*} \rightarrow 0$ (when $\mathrm{N}=4.9$ is held fixed). However, in this limit the bent-core structure of these molecule ceases to exist and reverts back to the rodlike uniaxial LCPs. Hence, we conclude that the $\mathbf{C H}$ phase is less favored in the phase transition sequence of 


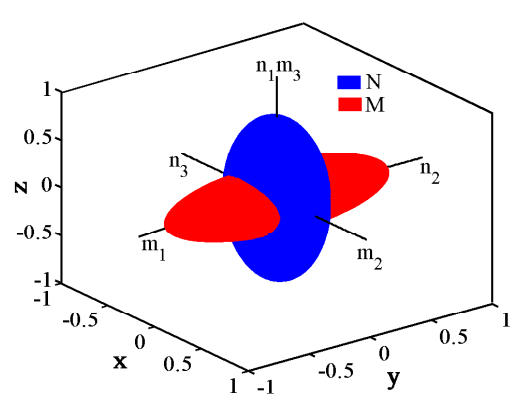

(a)

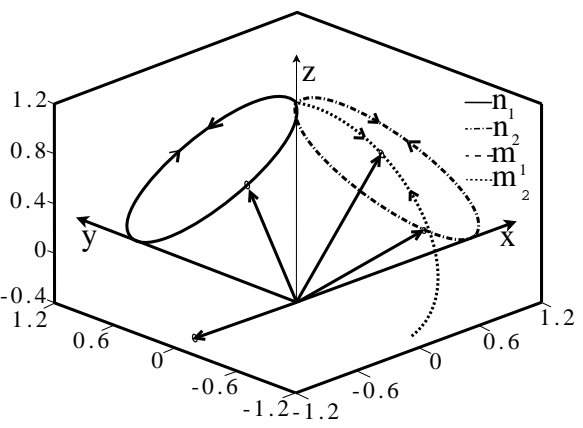

(c)

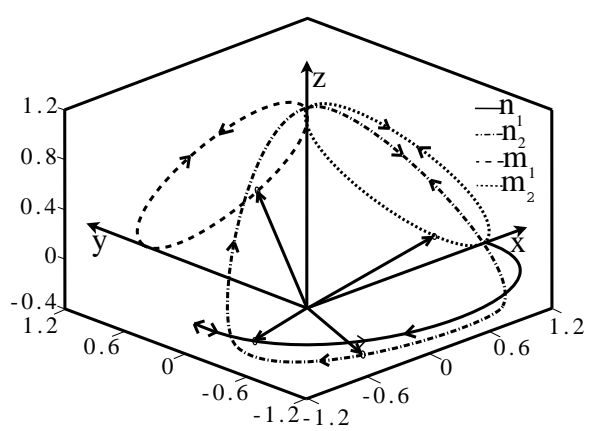

(e)

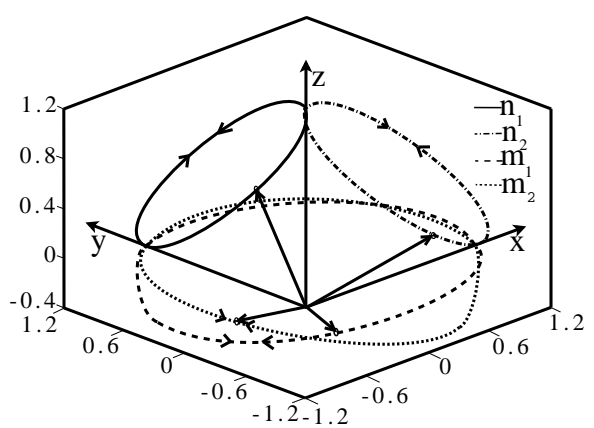

(b)

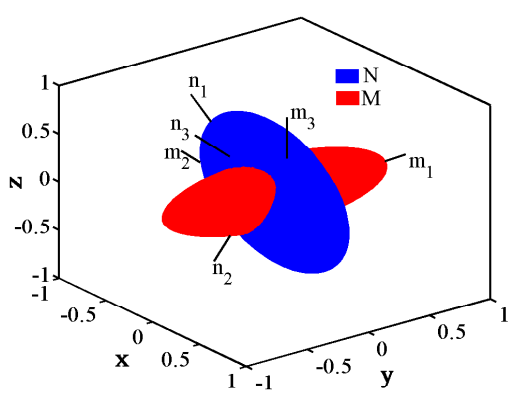

(d)

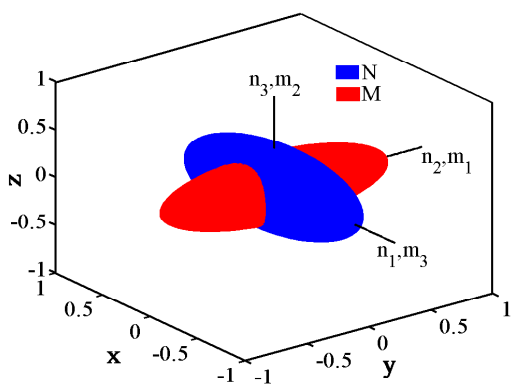

(f)

FIG. 4.1. Orientational phases at $\left(N=6.28, \beta^{*}=20^{\circ}\right)$. (i) Steady phases: (a) Log-Rolling phase (at $P e=1.0)$ (d) Out-of-Plane steady state (at Pe =7.0) (f) Flow-Aligning phase (at $P e=14.0$ ); and (ii) Periodic phases: (b) Mixed-Kayaking (at $P e=6.0$ with Period $T=5.02)$ (c) Wagging-Kayaking (at Pe $=8.0$, Period $T=4.90$ ) (e) Fluttering-Kayaking (at Pe $=10.0$ with Period $T=4.76)$

the biaxial phases of VLCPs as the bent angle increases compared to the case of the ellipsoidal biaxial LCP [33]. Further, we note that the largest eigenvalue selecting the major director $\mathbf{n}_{1}$ of $\mathbf{N}$ is larger than the one selecting the major director $\mathbf{m}_{1}$ of $\mathbf{M}$ for all the bent-angle values $\beta^{*}<45^{\circ}$. This trend reverses for $\beta^{*} \geq 45^{\circ}$, when suddenly the major director $\mathbf{m}_{1}$ of $\mathbf{M}$ becomes the distinguished VLCP mesoscale director. The sudden switching of the distinguished direction in the mesoscale ensemble is the major distinction in the kinetic theory of the shear-induced biaxial phases of the V-shaped 
LCP from the shear induced biaxial phases of ellipsoidal biaxial LCPs [33, 34]. Hence, the major director for the VLCP system is $\mathbf{n}_{1}$ at $\beta^{*}<45^{\circ}$ and $\mathbf{m}_{1}$ at $\beta^{*}>45^{\circ}$. We denote this major director collectively as $\tilde{\mathbf{m}}$ for all bent angles.

The steady Log-Rolling response $\mathbf{L R}$ is the one where $\tilde{\mathbf{m}}$ aligns along the vorticity (or in the fixed frame z-) direction. This alignment of $\tilde{\mathbf{m}}$ changes to the flow (or in the vicinity of the fixed frame $\mathrm{x}$-) direction in the steady Flow-Alignment response FA. The transitional Out-of-plane steady state $\mathbf{O S}$ is the one where $\tilde{\mathbf{m}}$ points away from the flow-velocity gradient plane but not in the vorticity direction either. Our numerical calculations reveal that roles of the eigenvector pairs: $\left(\mathbf{m}_{1}, \mathbf{m}_{2}\right)$ and $\left(\mathbf{n}_{1}, \mathbf{n}_{2}\right)$ reverse at $\beta^{*}=45^{\circ}$ in all the phases including the periodic motions. Below, we describe the trajectories of these eigenvectors in these motions at an arbitrarily fixed bent-angle and concentration (say $\beta^{*}=40^{\circ}, N=6.28$ ), as follows.

- The mixed-kayaking MK motion is a combination of a tilted kayaking motion of $\mathbf{m}_{1}$ and a full kayaking motion of $\mathbf{n}_{1}$ [33].

- The periodic fluttering-kayaking FK motion is a combination of wagging motion of $\mathbf{m}_{1}$ in the flow velocity-gradient plane (reminiscent of a fluttering motion of a falling leaf) and a completely non-circular (wagging) motion of $\mathbf{n}_{1}$.

- In the periodic wagging-kayaking $\mathbf{W K}$ motion, $\mathbf{m}_{1}$ and $\mathbf{m}_{2}$ undergo a tilted kayaking motion while osculating along the vorticity axis. The major direrctor $\mathbf{n}_{1}$ is held stationary while $\mathbf{n}_{2}$ wags along the (y-z) plane.

The second moments $\mathbf{M}$ and $\mathbf{N}$ in steady states and the principal and secondary eigenvectors of the second moments $\mathbf{M}, \mathbf{N}$ in time periodic motions (at the parameter values: $N=6.28, \beta^{*}=40^{\circ}$ ) are shown in figure 4.1, respectively. Again, we emphasize that the role of $\mathbf{M}$ and that of $\mathbf{N}$ reverses and so do their eigenvectors when $\beta^{*}>45^{\circ}$ in the above solutions.

4.2. Biaxial phases, order and rheology at $\beta^{*}=20^{\circ}$.

\begin{tabular}{|c|c|c|c|c|c|c|}
\hline$N$ & LR & MK & WK & OS & FK & FA \\
\hline 1.25 & - & - & - & - & - & $(0,14.0)$ \\
4.9 & $(0,4.3)$ & - & - & $(4.3,4.4)$ & - & $(4.4,14.0)$ \\
5.5 & $(0,4.3)$ & $(4.3,4.8)$ & $(4.8,5.4)$ & $(5.4,5.6)$ & $(5.6,8.0)$ & $(8.0,14.0)$ \\
6.28 & $(0,4.7)$ & $(4.7,5.2)$ & $(5.2,5.7)$ & $(5.7,5.9)$ & $(5.9,8.4)$ & $(8.4,14.0)$ \\
7.84 & $(0,5.7)$ & $(5.7,6.0)$ & $(6.0,6.4)$ & $(6.4,6.9)$ & $(6.9,8.9)$ & $(8.9,14.0)$ \\
\hline
\end{tabular}

TABLE 4.1. The shear strength windows corresponding to different orientational phases at $\beta^{*}=20^{\circ}$ and at the indicated values of $N$.

Table 4.1 presents the range of shear strength values in which the different orientational phases exist when the bent-angle is fixed at $\beta^{*}=20^{\circ}$ and the nematic concentration are given at the four specified values. The value of critical shear strength $\left(P e^{*}\right)$, at which the system transitions from one orientational phase to another, increases with the dimensionless concentration $(\mathrm{N})$. This is because the elastic repulsive torque due to the excluded volume potential (which opposes the torque due to the external flow) becomes stronger with increasing concentration. At low concentration $(N=4.9)$, the biaxial excluded volume interaction of these $\mathrm{V}$-shaped molecules, is not fully developed and hence the phase transitions occur only via the steady states while the periodic phases are absent. The steady state phases are LR at the small 
Peclet number range and FA in the high Peclet number range. The OS state exists in a narrow range of $P e$ at $N=4.9$ to connect the LR state on the left to the FA state on the right. When $N=5.5,6.28,7.84$, the solution of the Smoluchowski equation samples all the six phases mentioned previously. The phase transition sequence follows the pattern $\mathbf{L R} \rightarrow \mathbf{M K} \rightarrow \mathbf{W K} \rightarrow \mathbf{O S} \rightarrow \mathbf{F K} \rightarrow \mathbf{F A}$. The width of the internal in which OS exists widens as $N$ increases. Figure 4.2 presents the mesoscopic

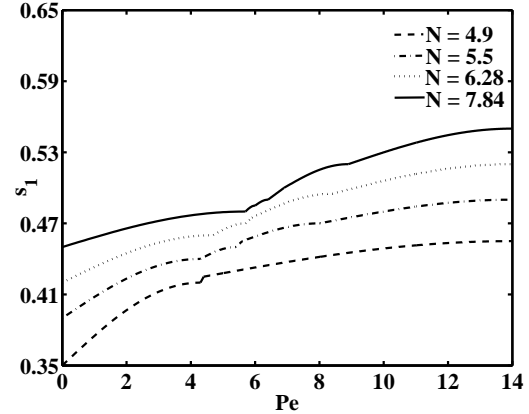

(a)

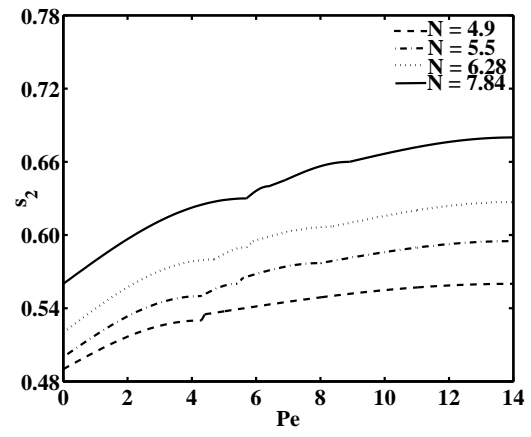

(c)

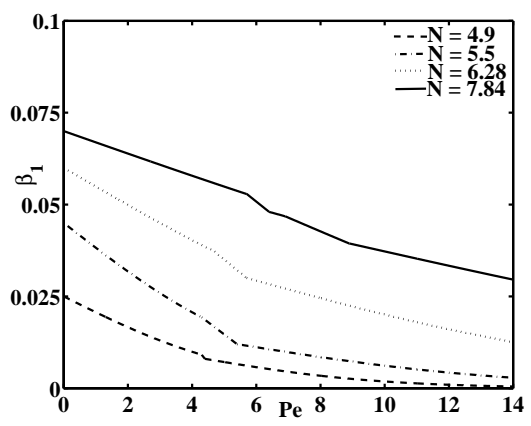

(b)

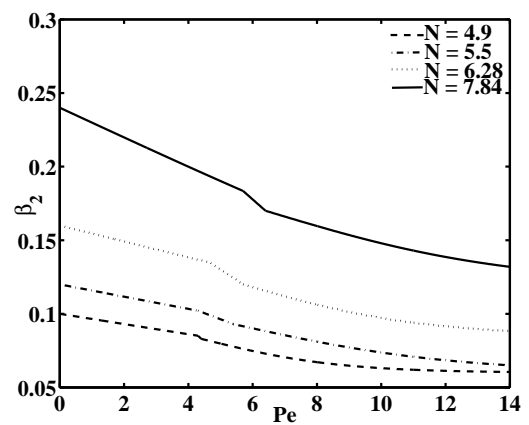

(d)

FIG. 4.2. Order Parameters: (a) $s_{1} ;$ (b) $\beta_{1} ;$ (c) $s_{2}$; (d) $\beta_{2}$ vs the non-dimensional shear strength (Pe) at the bent-angle $\beta^{*}=20^{\circ}$ and for different values of the nematic concentration $(N)$.

biaxial ordering via the order parameter information: $\left(s_{1}, \beta_{1}, s_{2}, \beta_{2}\right)$ at $\left(\beta^{*}=20^{\circ}\right)$. The order parameters are defined by

$$
s_{1}=\nu_{1}=\left\langle\left(\mathbf{m} \cdot \mathbf{m}_{1}\right)^{2}\right\rangle .
$$

$s_{1}$ measures the propensity of the axis of the arrow $\mathbf{m}$ to orient along the major director $\left(\mathbf{m}_{1}\right)$. The order parameter $\left(\beta_{1}\right)$, measures the difference of the nematic order of the molecular axis $\mathbf{m}$ with respect to the other two eigenvectors of $\mathbf{M}: \mathbf{m}_{2}, \mathbf{m}_{3}$, i.e.

$$
\beta_{1}=\left\langle\left(\mathbf{m} \cdot \mathbf{m}_{2}\right)^{2}\right\rangle-\left\langle\left(\mathbf{m} \cdot \mathbf{m}_{3}\right)^{2}\right\rangle .
$$

To measure the biaxiality due to the other two distinguished molecular axis $\mathbf{n}$ and $\mathbf{k}$, we label the axis as $\mathbf{Z}=\mathbf{m}_{1}$ and identify the eigen-vector corresponding to the largest eigenvalue of $\mathbf{N}$ and $\mathbf{K}$ as $Y$-axis. If this direction is not orthogonal to the $\mathbf{Z}$-axis, it is then projected onto the plane orthogonal to the $\mathrm{Z}$-axis and relabeled. 


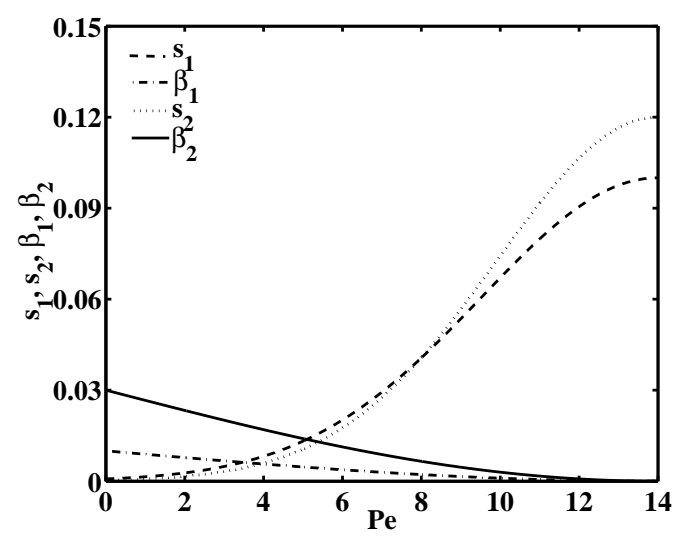

FIG. 4.3. Order parameters at $N=1.25$ and bent-angle $\beta^{*}=20^{\circ}$

The $\mathbf{X}$-axis is chosen to complete this to a right-handed system. The order parameter $\left(\beta_{2}\right)$ is defined as

$$
\begin{aligned}
\beta_{2} & =\frac{1}{2}\left(\mathbf{X}^{T} \cdot \mathbf{N} \cdot \mathbf{X}+\mathbf{Y}^{T} \cdot \mathbf{K} \cdot \mathbf{Y}-\mathbf{X}^{T} \cdot \mathbf{K} \cdot \mathbf{X}-\mathbf{Y}^{T} \cdot \mathbf{N} \cdot \mathbf{Y}\right) \\
& =\frac{1}{2}\left\langle(\mathbf{n} \cdot \mathbf{X})^{2}-(\mathbf{k} \cdot \mathbf{X})^{2}+(\mathbf{k} \cdot \mathbf{Y})^{2}-(\mathbf{n} \cdot \mathbf{Y})^{2}\right\rangle,
\end{aligned}
$$

which is the net difference between how well the molecular axes $\mathbf{n}$ and $\mathbf{k}$ are aligned with the $\mathbf{Y}$ and $\mathbf{X}$ direction respectively. If the molecular axes $\mathbf{n}$ and $\mathbf{k}$ are perfectly aligned with the $\mathbf{Y}$ and $\mathbf{X}$ directions respectively (or likewise the $\mathbf{X}$ and $\mathbf{Y}$ directions respectively), this quantity is 1 (or -1 ), while a zero value of this parameter shows that the system does not distinguish between any particular orientation in the plane orthogonal to Z. Clearly, it is the case when the molecule is uniaxial, where the probability distribution of $\mathbf{n}$ and $\mathbf{k}$ is identical. In other words, the choice of $\mathbf{n}$ and $\mathbf{k}$ is arbitrary so that $\beta_{2}$ must be zero then. Any nonzero value of $\beta_{2}$ must be generated by the departure of the molecular symmetry from uniaxiality. Finally, the order parameter $\left(s_{2}\right)$ gives another measure of intrinsic biaxiality. It is used to measure the net alignment of the molecular $\mathbf{m}$ and $\mathbf{n}$ axes along the $\mathbf{X}$ and $\mathbf{Y}$ direction respectively.

$$
\begin{aligned}
s_{2} & =\frac{1}{2}\left(\mathbf{X}^{T} \cdot \mathbf{M} \cdot \mathbf{X}+\mathbf{Y}^{T} \cdot \mathbf{N} \cdot \mathbf{Y}-\mathbf{X}^{T} \cdot \mathbf{N} \cdot \mathbf{X}-\mathbf{Y}^{T} \cdot \mathbf{M} \cdot \mathbf{Y}\right) \\
& =\frac{1}{2}\left\langle(\mathbf{m} \cdot \mathbf{X})^{2}-(\mathbf{n} \cdot \mathbf{X})^{2}+(\mathbf{n} \cdot \mathbf{Y})^{2}-(\mathbf{m} \cdot \mathbf{Y})^{2}\right\rangle .
\end{aligned}
$$

We observe that all the order parameters show a distinct second order discontinuity at the critical shear strength $\left(P e^{*}\right)$ where the phase transition occurs (refer to Table 4.1 for the values of $\left.P e^{*}\right)$ and that they are either monotonically increasing (parameters $s_{1}, s_{2}$ ) or decreasing (parameters $\beta_{1}, \beta_{2}$ ) vs. the non- dimensional shear strength. In general, they all increase with increasing LCP concentration. In the periodic phases (MK, WK, FK), the value of these order parameters (at a given shear strength) is averaged over one period. An increase in the shear-torque triggers an increase in the mesoscale order along the direction of the major director $\mathbf{m}_{1}$ (represented by the uniaxial parameters $s_{1}, s_{2}$ ), while simultaneously reducing the preferred 


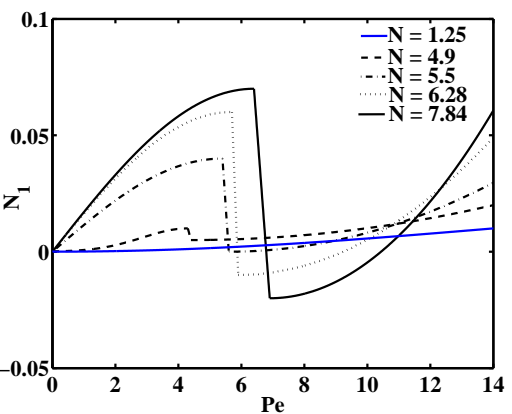

(a)

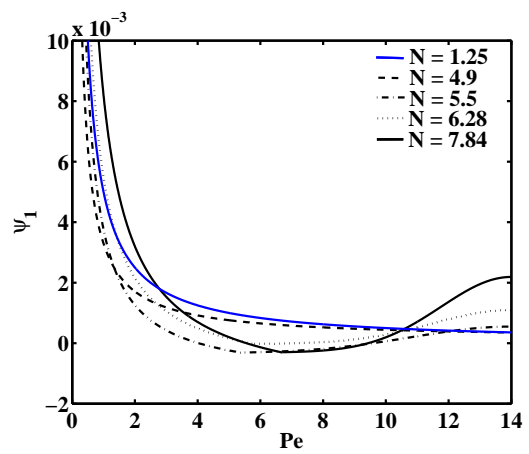

(c)

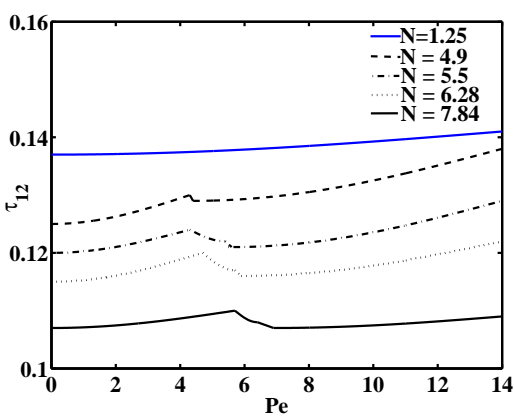

(e)

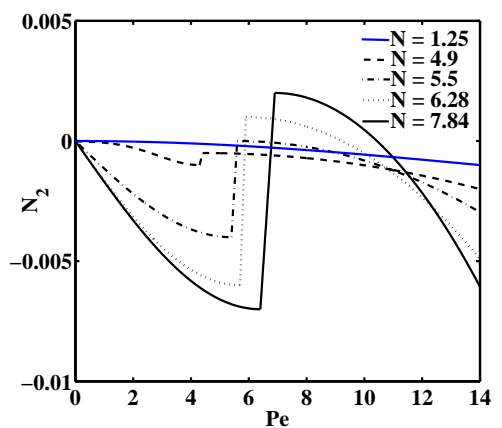

(b)

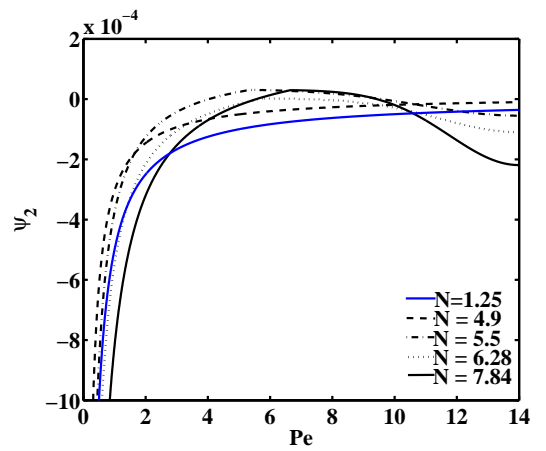

(d)

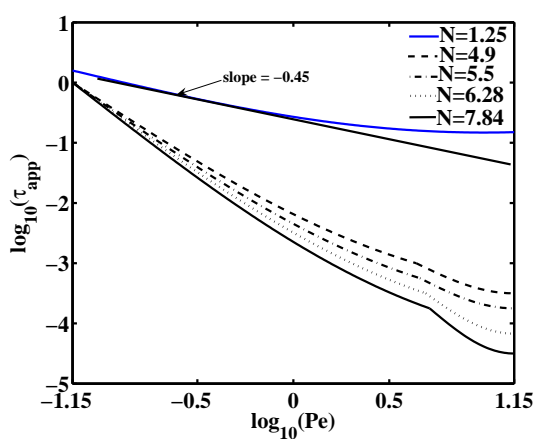

(f)

FIG. 4.4. Normalized rheometric functions vs Pe at four selected dimensionless concentration $N$ and the bent-angle $\beta^{*}=20^{\circ}$ : (a). the 1st normal stress difference $N_{1}$; (b) the 2nd normal stress difference $N_{2} ;(c)$ the $1^{\text {st }}$ normal stress coefficient $\psi_{1} ;(d)$ the $2^{\text {nd }}$ normal stress coefficient $\psi_{2}$; (e) the shear stress $\tau_{12} ;(f)$ the apparent viscosity: $\tau_{a p p} . N_{1}$ decays linearly in the steady OS-phase at a rate of: $-0.05(N=4.9),-0.2(N=5.5),-0.35(N=6.28)$ and $-0.33(N=7.84)$ respectively, while $\mathrm{N}_{2}$ increases at a rate of: $0.005(N=4.9), 0.02(N=5.5), 0.03(N=6.28,7.84)$ respectively. All the stress quantities are normalized with respect to $\nu k_{B} T$.

degree of orientation in a plane orthogonal to $\mathbf{m}_{1}$ (represented by the biaxiality parameter $\left.\beta_{2}\right)$. In the transitional OS-phase, the order parameters $\left(s_{1}, s_{2}\right)$ rise while the parameters $\left(\beta_{1}, \beta_{2}\right)$ decay along straight lines. The slopes of these straight lines of the respective order parameters in the OS-phase are 0.005 (for $s_{1}$ ), 0.005 (for $s_{2}$ ), -0.006 
(for $\beta_{1}$ ), and -0.002 (for $\beta_{2}$ ), and these slopes remain identical at all the chosen values of the concentrations. With varying LCP concentration, the order parameters range from (0.35-0.55) for $s_{1},(0.001-0.07)$ for $\beta_{1},(0.52-0.7)$ for $s_{2}$, and (0.006-0.24) for $\beta_{2}$. The small order parameter $\beta_{1}$ indicates a nearly uniaxial behavior in the molecular axis $\mathbf{m}$ in the mesoscopic ensemble.

In the case of $N=1.25$, it seems the only observable phase is the FA phase, in which the order parameters $s_{1,2}$ grow rapidly while the the other parameters $\beta_{1,2}$ decay. In this state, axis $\mathbf{m}$ tends to align in the direction of $\mathbf{Z}$ while the secondary axis $\mathbf{n}$ aligns in the direction of $\mathbf{Y}$. The overall nematic orders are low. Figure 4.3 depicts the four order parameters as functions of $P e \in[0,14]$ at $N=1.25$.

Figure 4.4 highlights rheological properties of the V-shaped molecules at $\beta^{*}=20^{\circ}$, where $R e=100, R e_{V L C P}=6$ are employed in all rheological calculations. Throughout the entire range of shear-strength studied, the first normal stress difference $\left(N_{1}=\right.$ $\left.\tau_{11}-\tau_{22}\right)$ remains one order of magnitude larger than the second normal stress difference $\left(N_{2}=\tau_{22}-\tau_{33}\right)$. In the low shear $(P e \leq 4.0)$ and the high shear regime $(P e \geq 12.0)$, the larger the nematic concentration $(\mathrm{N})$ is, the larger the magnitude of these stress differences is. Both the normal stress differences change signs $\left(N_{1}\right.$ from positive to negative and vice-versa for $N_{2}$ ) in the transitory OS-phase, which occurs in very narrow band of shear strength. Another change of sign (in both $N_{1}$ and $N_{2}$ ) occur in the periodic FK-phase. The two other time periodic phases MK, WK does not seem to induce any sign changes in the normal stress difference in the VLCP system and the behavior of their time-averaged normal stress difference follows those of LR and FA state.

At a fixed concentration $(N=5.5,6.28,7.84)$, the first normal stress coefficient $\left(\psi_{1}=\frac{N_{1}}{P e^{2}}\right)$ rapidly changes sign from positive at low shear $(P e \leq 5.4)$ to negative at intermediate shear $(5.4<P e \leq 7.0)$ before decaying to a small positive value in the high shear regime: $\mathrm{Pe}>7.0$ (vice-versa for $\psi_{2}=\frac{N_{2}}{P e^{2}}$ ). At $N=4.9$, however, $\psi_{1}$ remains positive $\left(\psi_{2}\right.$ remains negative) throughout the entire range of shear strength. The first and second normal stress difference also takes on opposite signs for the shear strength investigated. In general, the shear stress $\left(\tau_{12}\right)$ decreases with increasing particle concentration $(N)$. At a fixed value of $(N), \tau_{12}$ increases slightly in the $\mathbf{L R}$, MK, WK phases and then decays marginally in the other phases. The apparent viscosity $\left(\tau_{a p p}=\frac{\tau_{12}}{P e}\right)$ shows a drastic shear thinning behavior, of the order of $350 \%$ to $450 \%$, depending on the strength of the VLCP concentration. The rate of decay of the apparent viscosity varies with the different orientational phases. In general, the decay rate of the viscosity is inversely proportional to the concentration. At $N=1.25$, it's about -0.45 at small $P e \in[0,1]$.

4.3. Biaxial phases, order and rheology: the bent-angle effect. We next look into how the bent-angle $\beta^{*}$ affects phases and their transitions at $N=6.28$ and $N=1.25$, respectively.

The occurrence of the various orientational phases at different bent-angles $\left(\beta^{*}\right)$ and at a fixed strength of the excluded volume potential $N=6.28$ are tabulated in Table 4.2. Increasing the value of the bent-angle $\left(\beta^{*}\right)$ leads to an increase in the critical shear strength $\left(P e^{*}\right)$ of the biaxial phase transitions. This phenomena (also known as the "wing effect") is understood as follows: increasing the bent angle leads to a decrease in the length-to-breadth ratio of the bent-core molecules which in turn leads to a closer alignment of these molecules in a given mono-domain. The effect is similar to increasing the LCP concentration $(N)$, leading to a higher "particle density" in a mono-domain; a stronger torque due to the excluded volume, and hence 


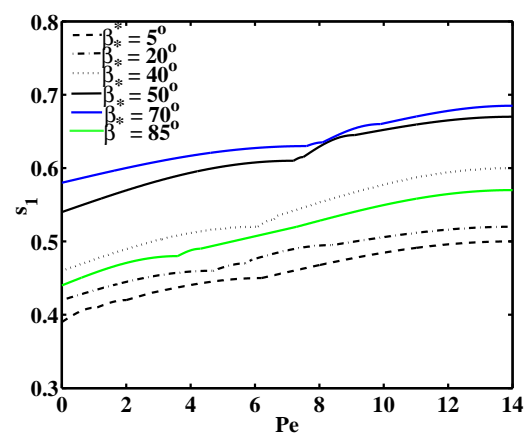

(a)

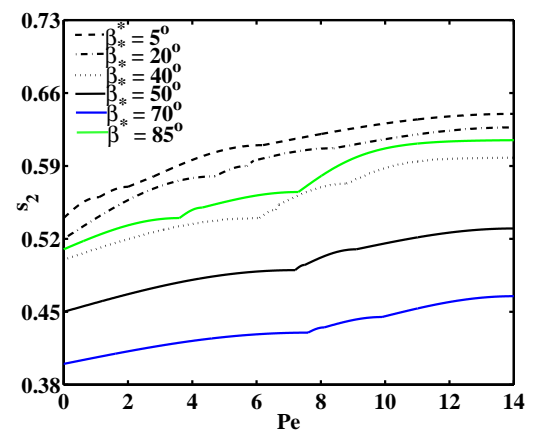

(c)

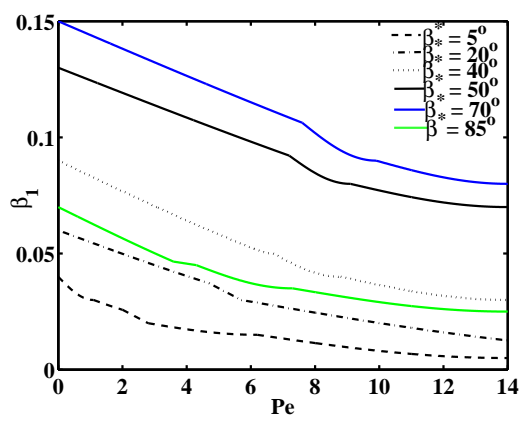

(b)

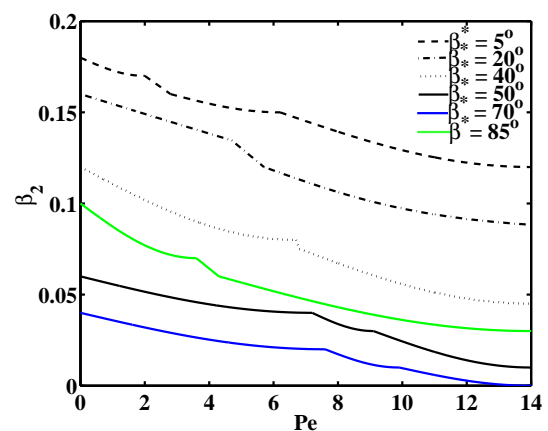

(d)

FIG. 4.5. Order Parameters: (a) $s_{1}$, (b) $\beta_{1}$, (c) $s_{2}$, and (d) $\beta_{2}$ versus the non-dimensional shear strength $(\mathrm{Pe})$ at the nematic concentration $N=6.28$ and a few selected values of the bent-angle $\left(\beta^{*}\right)$.

\begin{tabular}{|c|c|c|c|c|c|c|}
\hline$\beta^{*}$ & LR & MK & WK & OS & FK & FA \\
\hline $5^{\circ}$ & - & $(0,1.1)$ & $(1.1,2.0)$ & $(2.0,2.8)$ & $(2.8,6.2)$ & $(6.2,14.0)$ \\
$20^{\circ}$ & $(0,4.7)$ & $(4.7,5.2)$ & $(5.2,5.7)$ & $(5.7,5.9)$ & $(5.9,8.4)$ & $(8.4,14.0)$ \\
$40^{\circ}$ & $(0,6.1)$ & $(6.1,6.6)$ & $(6.6,7.0)$ & $(7.0,7.1)$ & $(7.1,8.9)$ & $(8.9,14.0)$ \\
$50^{\circ}$ & $(0,7.2)$ & $(7.2,7.5)$ & - & - & $(7.5,9.1)$ & $(9.1,14.0)$ \\
$70^{\circ}$ & $(0,7.6)$ & $(7.6,8.1)$ & - & - & $(8.1,9.9)$ & $(9.9,14.0)$ \\
$85^{\circ}$ & $(0,0.9)$ & $(0.9,2.3)$ & $(2.3,3.6)$ & $(3.6,4.3)$ & $(4.3,7.3)$ & $(7.3,14.0)$ \\
\hline
\end{tabular}

TABLE 4.2. The shear strength windows corresponding to the different orientational phases at $N=6.28$ and at the indicated values of $\beta^{*}$.

a higher value of critical shear is needed to overcome this repulsive torque. As the bent-angle $\beta^{*}$ is close to $90^{\circ}$, the critical transition values of Pe should go down as $\beta^{*}$ increases since the wing effect is reversed as $\beta^{*}$ approaches $90^{\circ}$. The introduction of the asymmetric flow breaks down the rotational symmetry of the VLCP ensemble. This fact is evident from the observation in table 4.2 that the nature of the phase transition at $\beta^{*}=\left(40^{\circ}, 50^{\circ}\right)$ or $\beta^{*}=\left(20^{\circ}, 70^{\circ}\right)$ (a complementary pair of bent-angles) is different. At $\beta^{*}=5^{\circ}, 20^{\circ}, 40^{\circ}$, the mesoscopic ensemble makes a second order transition in the phase sequence $\mathbf{W K} \rightarrow \mathbf{O S} \rightarrow \mathbf{F K}$ (refer to figure 4.12 ). At $\beta^{*}=50^{\circ}$, 


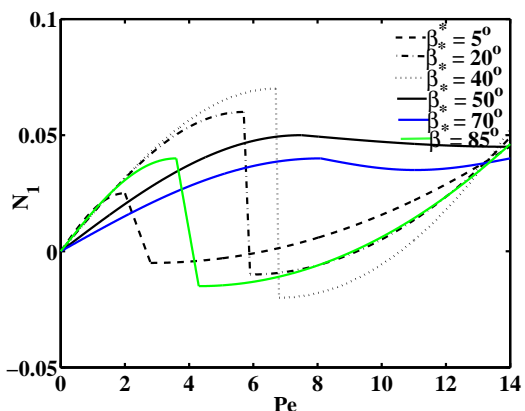

(a)

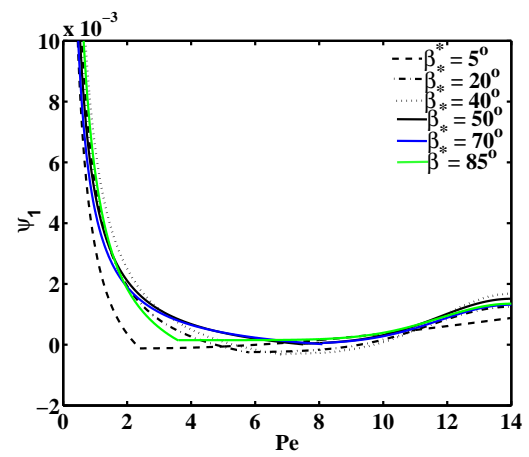

(c)

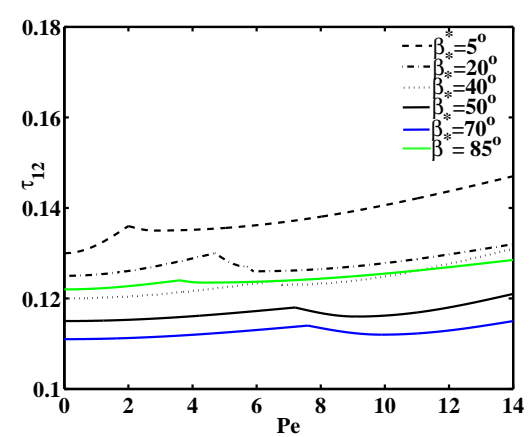

(e)

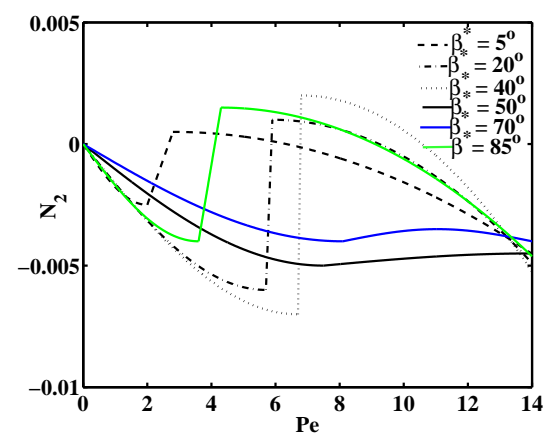

(b)

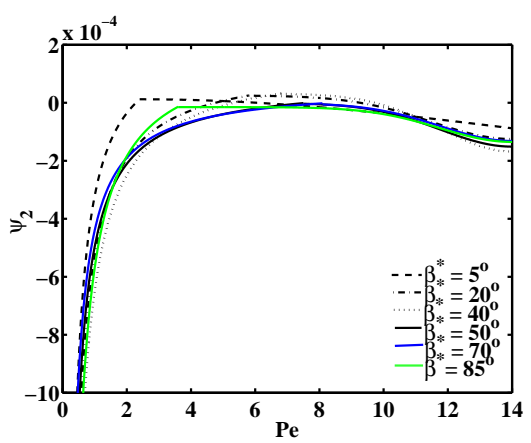

(d)

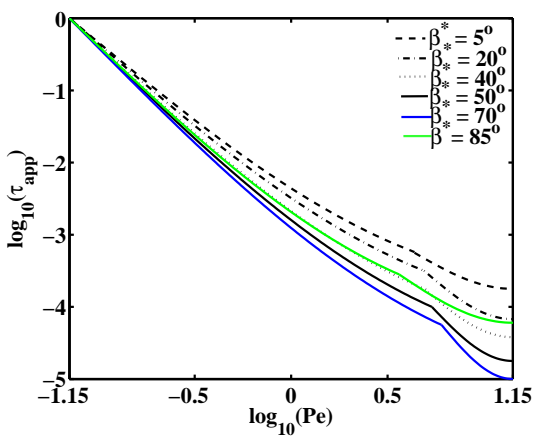

(f)

FIG. 4.6. Normalized rheometric functions vs non-dimensional shear strength (Pe) at concentration $N=6.28$ and a few different values of the bent-angle $\beta^{*}:$ (a) the $1^{\text {st }}$ normal stress difference $N_{1}$; (b) the $2^{\text {nd }}$ normal stress difference $N_{2} ;$ (c) the $1^{\text {st }}$ normal stress coefficient $\psi_{1}$; (d) the $2^{\text {nd }}$ normal stress coefficient $\psi_{2} ;(e)$ the shear stress $\tau_{12} ;(f)$ the apparent viscosity: $\tau_{a p p} . N_{1}$ decays linearly in the steady OS-phase at a rate of: $-0.04\left(\beta^{*}=5^{\circ}\right),-0.35\left(\beta^{*}=20^{\circ}\right)$ and $-0.9\left(\beta^{*}=40^{\circ}\right)$ respectively, while $N_{2}$ increases at a rate of: $0.004\left(\beta^{*}=5^{\circ}\right), 0.03\left(\beta^{*}=20^{\circ}\right)$ and $0.09\left(\beta^{*}=40^{\circ}\right)$ respectively. All the plotted stress quantities are normalized with respect to $\nu k_{B} T$.

however, the nature of this phase transition changes into a first order transition at $P e^{*}=7.5: \mathbf{M K} \rightarrow \mathbf{F K}$. When the bent-angle is increased to $\beta^{*}=70^{\circ}$, this first order transition occurs at $P e^{*}=8.1$. The nature of the phase transition versus the bent- 


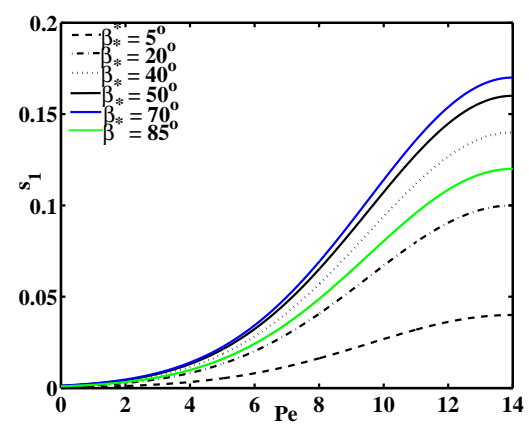

(a)

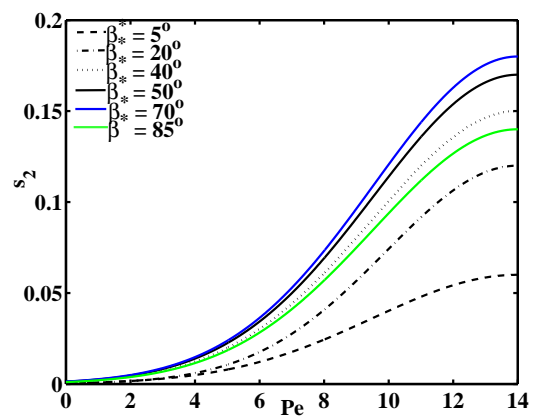

(c)

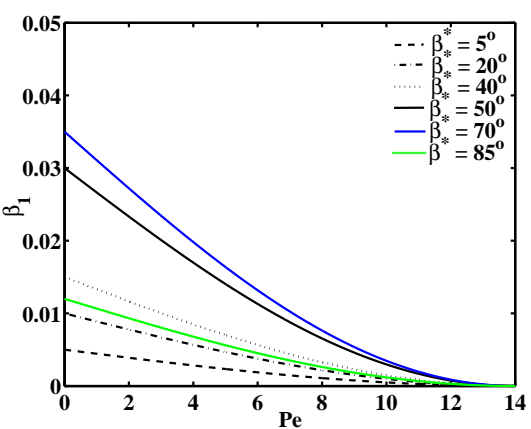

(b)

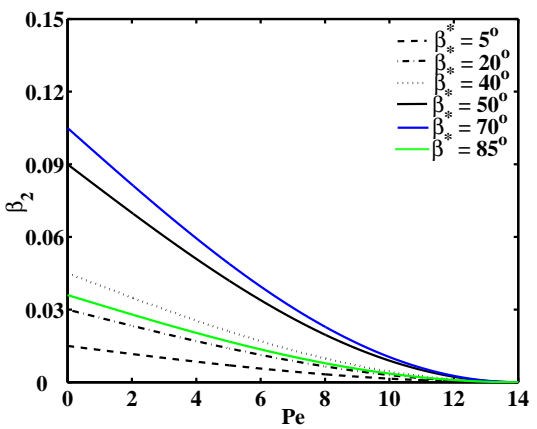

(d)

FIG. 4.7. Order Parameters: (a) $s_{1}$, (b) $\beta_{1}$, (c) $s_{2}$, and (d) $\beta_{2}$ versus the non-dimensional shear strength $(P e)$ at the nematic concentration $N=1.25$ and selected values of the bent-angle $\left(\beta^{*}\right)$.

angle will be analyzed in more detail in the next section. The apparent absence of the LR state at small bent-angle is reminiscent of the rodlike LCPs at intermediate shear strength. The first order phase transition at large bent-angles also draws analogies to the ellipsoidal biaxial LCPs under shear [33, 34]. The order parameters versus the bent-angles at $N=6.28$ are presented in figure 4.5 . The salient features observed throughout these graphs are:

- the presence of a second order (continuous but not differentiable) transition of these order parameters at the critical shear strength $\left(P e^{*}\right)$,

- order parameters $\left(s_{1}, s_{2}\right)$ increase when the shear strength increases and the bent-angle is held constant, whereas $\left(\beta_{1}, \beta_{2}\right)$ decrease as $P e$ increases, and

- increasing the bent-angle marks an increase in the parameters $\left(s_{1}, \beta_{1}\right)$ and decrease in $\left(s_{2}, \beta_{2}\right)$ decreases.

By definition $\left(s_{1}\right)$ is proportional to the eigenvalue of the second moment $\mathbf{M}$, while $\beta_{2}$ measures the "net" projection of the second moments $\mathbf{N}, \mathbf{K}$ on a specific plane. A rising value of $s_{1}, \beta_{1}$ (and a decaying value of $s_{2}, \beta_{2}$ ) indicates that in general the eigenvalues $\mathbf{M}$ get larger than the eigenvalues of $\mathbf{N}$ and $\mathbf{M}$ shows more biaxiality at larger values of $\beta^{*}$. Hence, it is not surprising that the major director of the VLCP ensemble switches from $\mathbf{m}_{1}$ to $\mathbf{n}_{1}$ at $\beta^{*}=45^{\circ}$. The range of these parameters vary from $(0.39-0.68)$ for $s_{1},(0.005-0.15)$ for $\beta_{1},(0.42-0.66)$ for $s_{2}$, and $(0.0-0.18)$ for $\beta_{2}$. 


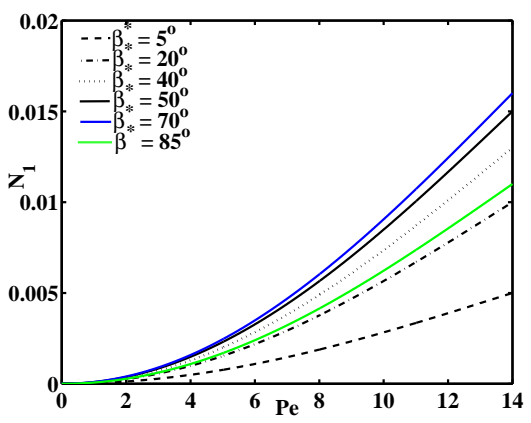

(a)

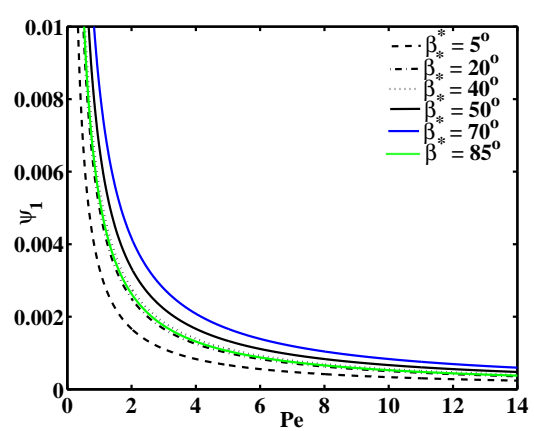

(c)

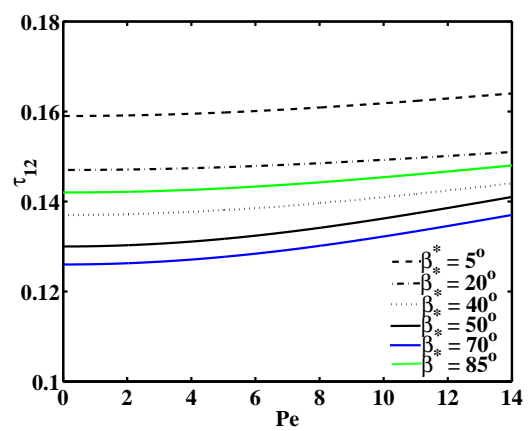

(e)

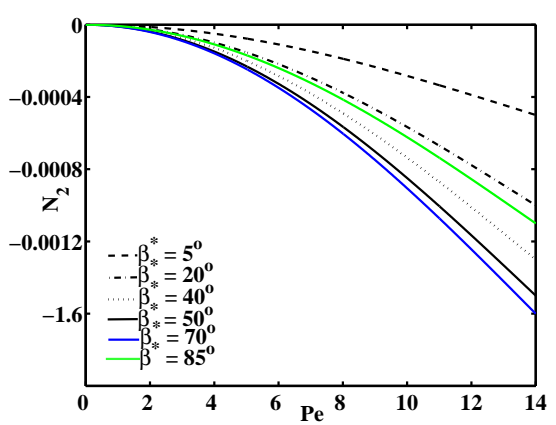

(b)

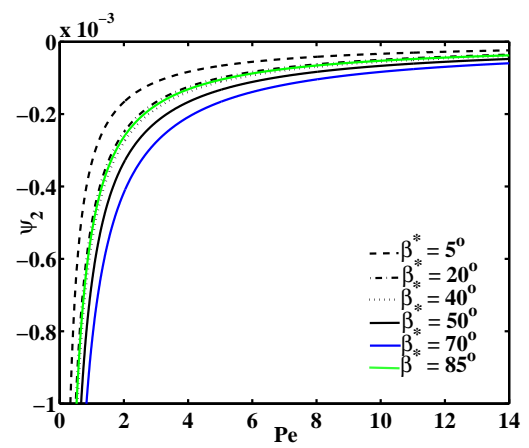

(d)

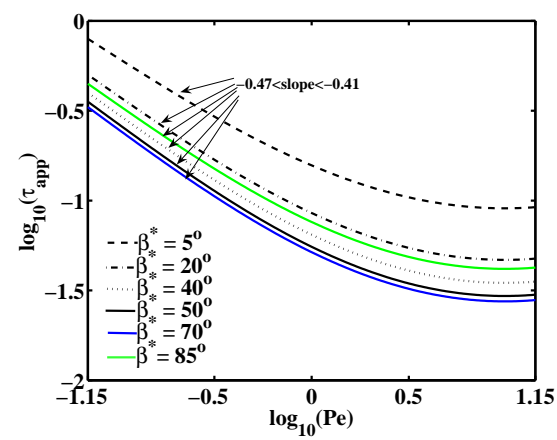

(f)

FIG. 4.8. Normalized rheometric functions vs non-dimensional shear strength (Pe) at concentration $N=6.28$ and a few different values of the bent-angle $\beta^{*}:$ (a) the $1^{\text {st }}$ normal stress difference $N_{1} ;(b)$ the $2^{\text {nd }}$ normal stress difference $N_{2} ;$ (c) the $1^{\text {st }}$ normal stress coefficient $\psi_{1} ;(d)$ the $2^{\text {nd }}$ normal stress coefficient $\psi_{2}$; (e) the shear stress $\tau_{12} ;(f)$ the apparent viscosity: $\tau_{a p p}$.

Figure 4.6 depicts the rheological measures of the ensemble of V-shaped molecules at $N=6.28$. Unlike the case of varying nematic concentration, the maximal magnitude of the normal stress differences starts to decrease at higher values of the bentangle $\left(\beta^{*}>45^{\circ}\right)$, although $N_{1}$ still retains one order of magnitude larger than $N_{2}$ throughout the entire range of the shear strength studied. By changing the molecular bent-angle we change the major director of the mesoscale ensemble, which leads to a 


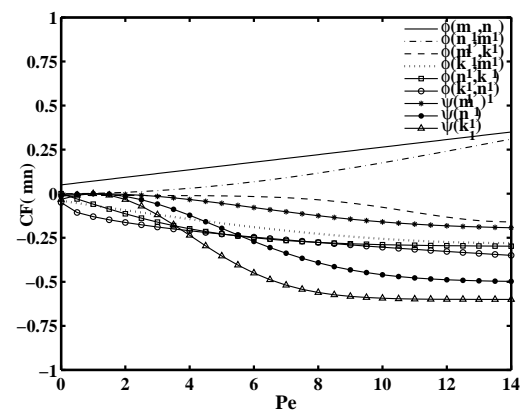

(a)

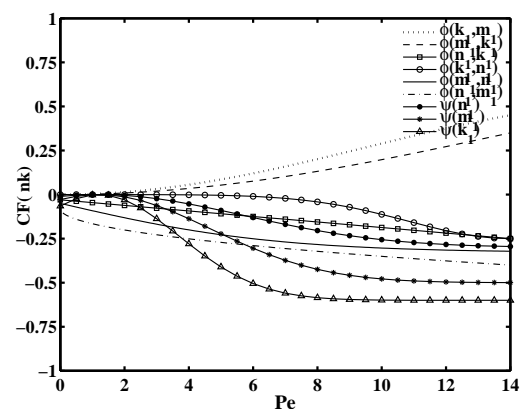

(b)

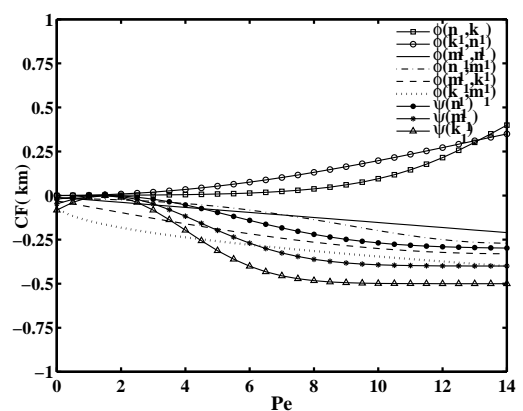

(c)

FIG. 4.9. Orientational Correlation Functions of $(a)\langle\mathbf{m n}\rangle,(b)\langle\mathbf{n k}\rangle$, and $(c)\langle\mathbf{k m}\rangle$ at the parameter values: $\left(N=4.9, \beta^{*}=20^{\circ}\right)$. The meso-structure undergoes the transition $\mathbf{L R} \rightarrow \mathbf{O S} \rightarrow \mathbf{F A}$ at $P e^{*}=4.3,4.4$, respectively.

significant change in the rheological properties including the stress differences. The normal stress coefficient $\left(\psi_{1}\right)$ also changes sign from positive to negative in the low shear-strength region: $0 \leq P e \leq 5.7$, and back to positive in the high shear regime: $\mathrm{Pe}>10.0$ (vice-versa for $\psi_{2}$ ). The shear stress $\left(\tau_{12}\right)$ decreases with increasing bentangle and for a fixed $\beta^{*}$, and it achieves a maximum at the end of the WK-phase $\left(\right.$ at $\beta^{*}=5^{\circ}, 20^{\circ}, 40^{\circ}$ ) and at the end of the MK-phase (at $\left.\beta^{*}=50^{\circ}, 70^{\circ}\right)$. Similar to the case in section 4.2 , the apparent viscosity $\left(\tau_{\text {app }}\right)$ shows shear thinning, at a rate which decreases with the bent-angle, another ramification of the wing effect.

At $N=1.25$, the phase is clearly biaxial for all bent-angles listed. All four order parameters are positive. The order parameters increase with respect to the bent angle to $70^{\circ}$ and decay afterwards at $\beta^{*}=85^{\circ}$ (shown in figure 4.7). The normal stress difference, shear stress, and viscosity all follow an analogous trend as the bent angle increases. The slops at all angles fall within $[-0.47,-0.41]$ (figure 4.8), which are in the approximity of the experimental results for the nematic phases of bent-core biaxial liquid crystals [3].

4.4. Correlation matrices. In this section, we describe the biaxial order of the VLCPs using projection of the mixed moments $(\langle\mathbf{m n}\rangle,\langle\mathbf{n k}\rangle,\langle\mathbf{k m}\rangle)$ onto the plane spanned by the major directors $\left(\mathbf{m}_{1}, \mathbf{n}_{1}, \mathbf{k}_{1}\right)$, or the Orientational Correlation Functions (OCF) and Auto correlation functions (ACF). The bent-angle is fixed at $\beta^{*}=20^{\circ}$ and the nematic concentration takes on the values $\mathrm{N}=4.9,5.5,6.28,7.84$, 


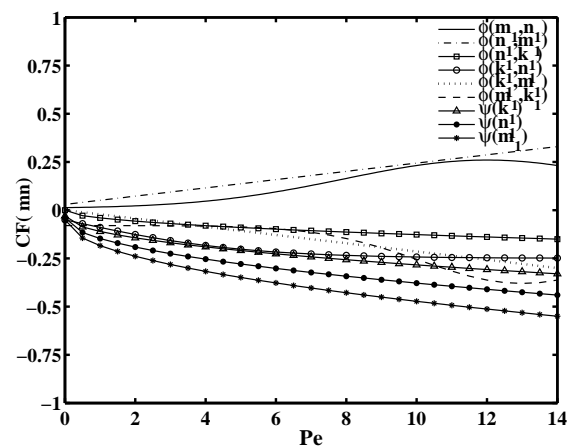

(a)

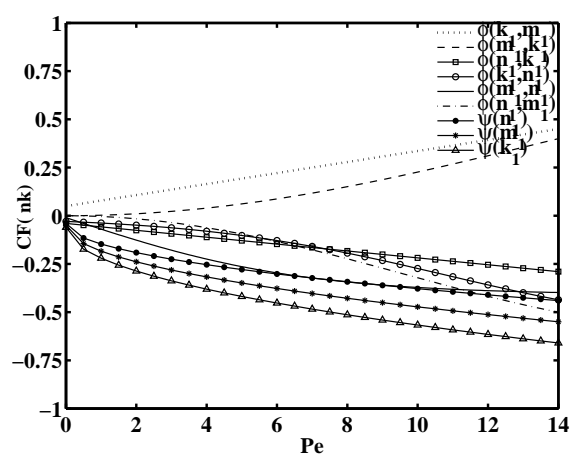

(b)

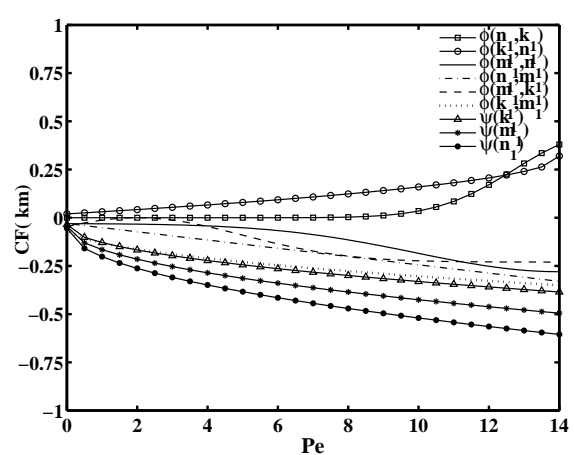

(c)

FIG. 4.10. Orientational Correlation Functions of $(a)\langle\mathbf{m n}\rangle,(b)\langle\mathbf{n k}\rangle$, and $(c)\langle\mathbf{k m}\rangle$ at the parameter values: $\left(N=5.5, \beta^{*}=20^{\circ}\right)$. The meso-structure undergoes the transition $\mathbf{L R} \rightarrow \mathbf{M K} \rightarrow \mathbf{W K} \rightarrow \mathbf{O S} \rightarrow \mathbf{F K} \rightarrow \mathbf{F A}$ at $P e^{*}=4.3,4.8,5.4,5.6,8.0$, respectively.

respectively.

The OCF matrices at $N=4.9$ are presented in figure 4.9. At equilibrium, the OCF's are zero (or nearly zero). We note from our numerical experiments that the highest value of the entries of the mixed moments at $P e=0.0$ is of the order of $10^{-3}$. We also notice that when we increase the order of the spectral accuracy from $L_{0}=10$ to $L_{0}=20$, the highest value of these entries drops to $10^{-5}$ [33]. Hence, we attribute the non-zero value of these entries at $P e=0.0$ due to the numerical error of the spectral approximation and conclude that in the ideal situation $\left(L_{0}=\infty\right)$ these will be identically zero. A zero value of the mixed moments suggests that the second moments $(\mathbf{M}, \mathbf{N}, \mathbf{K})$ share the same eigen-frame at equilibrium. As the flow is turned on the OCF's diverge away from the zero values. The maximum range of admissible values of the OCFs lie within $(-1,1)$. At $N=4.9$ and $P e=14.0$, however, the OCFs lie in the range $(-0.5,0.5)$ and hence cover only $50 \%$ of the maximum range of admissible values. This suggests that there is only a partially enhanced order of the molecular axis $(\mathbf{m}, \mathbf{n}, \mathbf{k})$ in sheared kinetics. One noticeable feature among the OCFs is that the ACFs are all negative, $0>\psi^{m n}\left(\mathbf{m}_{1}\right)>\psi^{m n}\left(\mathbf{n}_{1}\right)>\psi^{m n}\left(\mathbf{k}_{1}\right)$, the order of $\psi^{n k}\left(\mathbf{m}_{1}\right)$ and $\psi^{n k}\left(\mathbf{n}_{1}\right)$ switches, $\psi^{n k}\left(\mathbf{m}_{1}\right)<\psi^{n k}\left(\mathbf{n}_{1}\right)$, and it maintains the same order in the OCFs of ( $\mathbf{m k})$.

In figure 4.10, we highlight the OCF's at $N=5.5$. Again, the OCFs diverge away 


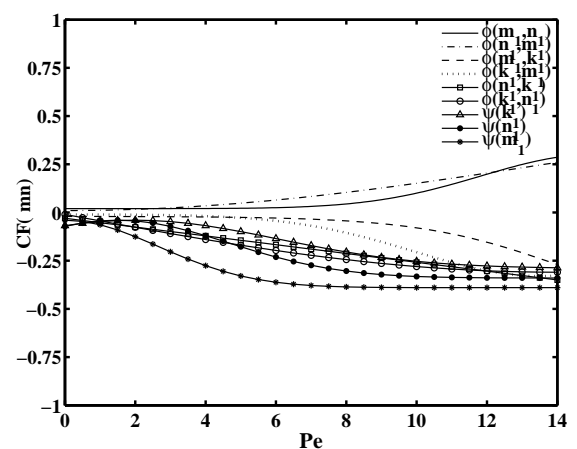

(a)

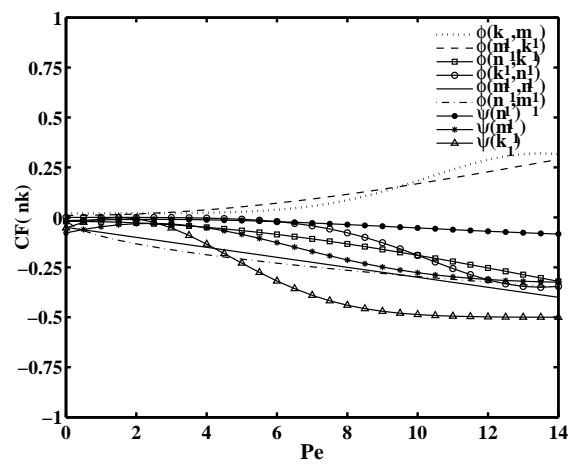

(b)

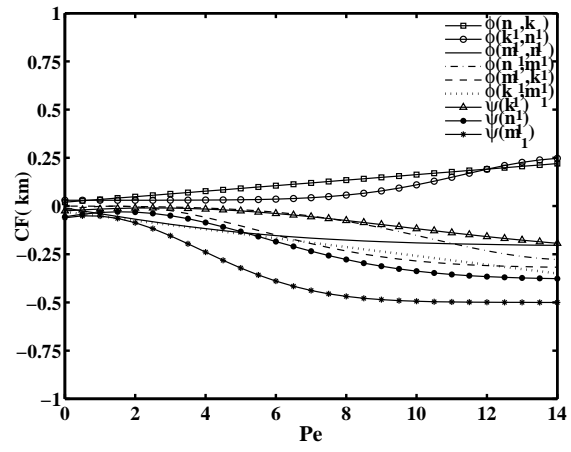

(c)

FIG. 4.11. Orientational Correlation Functions of $(a)\langle\mathbf{m n}\rangle, \quad(b)\langle\mathbf{n k}\rangle$, and $(c)\langle\mathbf{k m}\rangle$ at the parameter values: $\left(N=7.84, \beta^{*}=20^{\circ}\right)$. The meso-structure makes the transition $\mathbf{L R} \rightarrow \mathbf{M K} \rightarrow \mathbf{W K} \rightarrow \mathbf{O S} \rightarrow \mathbf{F K} \rightarrow \mathbf{F A}$ at $P e^{*}=5.7,6.0,6.4,6.9,8.9$, respectively.

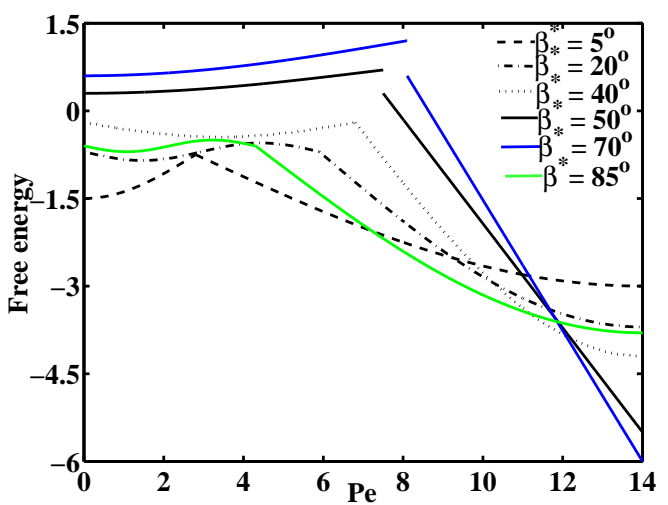

FIG. 4.12. Free energy density normalized by $\nu k_{B} T$ at $N=6.28$ and six selected bent-angles. 
from their zero (or nearly zero) equilibrium values at $P e=0.0$, to some values at $P e=14.0$ which lie in the approximate range $-0.75 \leq \mathrm{OCF} \leq 0.5$. The orders of the ACF with respect to $\mathbf{m n}$ and $\mathbf{m k}$ completely reverse those in the case of $N=4.9$ while the same ordering remains in the ACFs with respect to $\mathbf{n k}$. The case of $N=6.28$ is analogous to the case of $N=5.5$. At $N=7.84$, however, the ordering in $\psi^{k m}$ goes like $\psi^{k m}\left(\mathbf{m}_{1}\right)<\psi^{k m}\left(\mathbf{n}_{1}\right)<\psi^{k m}\left(\mathbf{k}_{1}\right)$. Figure 4.11 highlights the correlation function information of the mixed moments at $N=7.84$. It shows that the ordering of the molecular axes $(\mathbf{m}, \mathbf{n}, \mathbf{k})$ with respect to the major directors $\left(\mathbf{m}_{1}, \mathbf{n}_{1}, \mathbf{k}_{1}\right)$ sensitively depends on the strength of the torque due to the excluded volume potential (which depends on the strength of the nematic concentration $N$ ).

4.5. Phase transition phenomena: a perspective from the free energy consideration. Figure 4.12 presents the time-averaged "free-energy" density of the $\mathrm{V}$-shaped molecules at $\mathrm{N}=6.28$ and for bent-angle values $\beta^{*}=5^{\circ}, 20^{\circ}, 40^{\circ}, 50^{\circ}, 70^{\circ}$, respectively. The major observations are:

- (a) At $\beta^{*}=50^{\circ}$, the steady OS-phase is absent from the sequence of phase transitions (refer to table 4.2). The nature of the phase transition changes from second order (at $\beta^{*}=5^{\circ}, 20^{\circ}, 40^{\circ}$ ) to first order at $\beta^{*}=50^{\circ}$. Clearly, there exists a critical bent-angle $40^{\circ} \leq \beta^{*} \leq 50^{\circ}$, at which there is a change in the nature of the phase transition from the $2^{\text {nd }}$ order $\leftrightarrow$ the $1^{\text {st }}$ order. The exact value of this critical bent-angle has yet been determined.

- (b) At low shear, the free energy increases with increasing value of the bentangles. However, at high shear, this trend reverses. A possible reasoning by Johnson et. $e l[22]$ in the numerical simulations of a cluster of VLCPs with Gay-Berne potential is as follows: at low shear, a higher value of bentangle, lowers the "length-to-breadth" ratio of the V-shaped molecules, which in turn increases the "packing density" of these molecules in a given monodomain. This increase in "packing density" leads to a physically unfavorable configuration, and hence a higher free-energy density. This is also consistent with the notion that the strength of the interaction potential increases as alluded to earlier. A very high value of shear somewhat negates this "packing frustration", and aligns these molecules in a configuration which becomes increasingly favorable with higher bent-angles.

\section{Conclusion}

We have presented a fully coupled hydrodynamic theory for the solution of bentcore or V-shaped biaxial liquid crystal polymers. The theory is formulated as a kinetic theory, where the dominating excluded volume interaction among the bent-core molecules and the flow induced kinematics on the bent-core molecules are accounted for. The extra elastic stress is derived using a virtual work principle while the viscous stress is calculated from an approximate dissipation functional. We developed a Galerkin-Spectral code to integrate the Smoluchowski equation using a Wigner function basis in the monodomain. Limited in biaxial phases, we investigated the flow induced phases at various concentrations and bent-angles of the molecule as well as shear induced phase transitions. Three truly biaxial steady states and three time dependent motions/phases are observed in the computations. The chaotic motion, known in rigid uniaxial liquid crystal polymers is apparently elusive in the bent-core biaxial liquid crystal polymer at moderate bent-angles, indicating the enhanced biaxiality and asymmetry in the bent-core molecular configuration somehow impacts on the erratic time-dependent motion and makes it unfavorable in the mesoscopic 
ensemble. Rheological studies demonstrate consistent shear shinning behavior in the mesoscopic system, positive first normal stress difference in the steady Logrolling and Flow-Aligning phase. The first normal stress and the second normal stress difference can change signs while going through the transitional Out-of-Plane steady state and a time-periodic phase. The first normal stress difference can be positive in some time-periodic phases known as MK and WK as well. At the regime of the weak intermolecular potential, the slope of the apparent viscosity predicted by this theory is in the approximity of the experimental data on a couple of bent-core biaxial liquid crystals [3].

Acknowledgment. Qi Wang's research is partially supported by the Air Force Office of Scientific Research, Air Force Materials Command, USAF, under grant number FA9550-08-1-0107 and the National Science Foundation through grants DMS0605029, DMS-0626180, DMS-0724273, DMS-0819051, and DMS-0908330.

\section{REFERENCES}

[1] B.R. Acharya, A. Primak and S. Kumar, Biaxial nematic phase in bent-core thermotropic mesogens, Phys. Rev. Lett., 92, 145506, 2004.

[2] M.P. Allen, Computer simulation of a biaxial liquid crystal, Liq. Cryst., 8, 499-511, 1990.

[3] C. Bailey, Structure and Rheology of Some Bent Core Liquid Crystals, Ph. D. Thesis, Kent State University, 2008.

[4] V. Berjnov, V. Cabuil, R. Perzynski and Y. Raikher, Lyotropic system potassium laurate/1-decanol/water as a carrier medium for a ferronematic liquid crystal: phase diagram study, J. Phys. Chem. B, 102, 7132, 1998.

[5] R. Bernardi and C. Zannoni, Do thermotropic biaxial nematics exist? A Monte Carlo study of biaxial Gay-Berne particles, J. Chem. Phys., 113, 5971-5979, 2000.

[6] F. Biscarini, C. Chiccoli, P. Pasini, F. Semeria and C. Zannoni, Phase diagram and orientational order in a biaxial lattice model: a Monte Carlo study, Phys. Rev. Lett., 75, 1803-1806, 1995.

[7] F. Bisi, E.G. Virga, E.C. Gartland, G. De Matteis, A.M. Sonnet and G.E. Durand, Universal mean-field phase diagram for biaxial nematics obtained from a minimax principle, Phys. Rev. E, 73, 051709, 2006.

[8] M.C. Calderer, M.G. Forest, and Q. Wang, Kinetic theories and mesoscopic models for solutions of nonhomogeneous liquid crystal polymers, J. of Non-Newtonian Fluid Mech., 120, 69, 2004.

[9] T. Carlsson and F. Leslie, Behaviour of biaxial nematics in the presence of electric and magnetic fields: evidence of bistability, Liq. Cryst., 10, 325-340, 1991.

[10] C. Chiccoli, P. Pasini, F. Semeria and C. Zannoni, Lecture Notes in Computer Science, 2110, 555-560, 2001.

[11] G. De Matteis, S. Romano and E. Virga, Bifurcation analysis and computer simulation of biaxial liquid crystals, Phys. Rev. E., 72, 04170, 2005.

[12] M. Doi and S.F. Edwards, Theory of Polymer Dynamics, Clarendon Press, Oxford, 1986.

[13] B.J. Edwards, D.J. Keffer and C.W. Reneau, An examination of the shear-thickening behavior of high-molecular-weight polymers dissolved in low-viscosity Newtonian solvents, J. App. Polymer Phys., 85, 1714, 2002.

[14] J.W. Emsley, Nuclear Magnetic Resonance of Liquid Crystals, 1985.

[15] J. Feng, G. Sgalari and L.G. Leal, A theory for flowing nematic polymers with orientational distortions, J. Rheol., 44, 1085-1101, 2000.

[16] J.L. Figyeirinhas, C. Cruz, D. Filip, G. Feio, A.C. Ribeiro, Y. Frere, T. Meyer and G.H. Mehl, Deuterium NMR investigation of the biaxial nematic phase in an organosiloxane tetrapode, Phys. Rev. Lett., 94, 107802, 2005.

[17] A.A. De Melo Filho, A. Laverde and F.Y. Fujiwara, Observation of two biaxial nematic mesophases in the tetradecyltrimethylammonium bromide/decanol/water system, Langmuir, 19, 1127-1132, 2003.

[18] M.G. Forest, Q. Wang and R.Zhou, The weak shear kinetic phase diagram for nematic polymers, Rheol. Acta, 43, 17-37, 2004

[19] M.G. Forest, Q. Wang and R.Zhou, The flow-phase diagram of Doi-Hess theory for sheared 
nematic polymers II: finite shear rates, Rheol. Acta, 44, 80-93, 2004.

[20] M.G. Forest, S. Sircar, Q. Wang and R. Zhou, Monodomain dynamics for rigid rod and platelet suspensions in strongly coupled coplanar linear flow and magnetic fields. II. Kinetic theory, Phys. Fluids, 18, 103102, 2006.

[21] M.J. Freiser, Ordered states of a nematic liquid, Phys. Rev. Lett., 24, 19, 1041, 1970.

[22] S.J. Johnston, R.J. Low and M.P. Neal, Computer simulation of apolar bent-core and rodlike molecules, Phys. Rev. E, 65, 051706, 2002.

[23] L. Longa, P. Grzybowski, S. Romano and E.G. Virga, Minimal coupling model of the biaxial nematic phase, Phys. Rev. E, 71, 051714, 2005.

[24] F.M. Leslie, Theory of flow phenomena in liquid crystals, Advances in Liquid Crystals, 4, $1-81,1979$.

[25] T.C. Lubensky and L. Radzihovsky, Theory of bent-core liquid-crystal phases and phase transitions, Phys. Rev. E, 66, 031704, 2002.

[26] G.R. Luckhurst, Liquid crystals: A missing phase found at last? Nature (London), 430, 413, 2004.

[27] L. A. Madsen, T.J. Dingemans, M. Nakata and E.T. Samulski, Thermotropic biaxial nematic liquid crystals, Phys. Rev. Lett., 92, 145505, 2004.

[28] K. Merkel, A. Kocot, J.K. Vij, R. Korlacki, G.H. Mehl and T. Meyer, Thermotropic biaxial nematic phase in liquid crystalline organo-siloxane tetrapodes, Phys. Rev. Lett., 93, 237801, 2004.

[29] P.L. Nordio, G. Rigatti and U. Segre, Dielectric relaxation theory in nematic liquids, Molec. Phys., 25, 129-136, 1973.

[30] J. Pelaez and M. Wilson, Atomistic simulations of a thermotropic biaxial liquid crystal, Phys. Rev. Lett., 97, 267801, 2006.

[31] M.E. Rose, Elementary Theory of Angular Momentum, Wiley, 1957.

[32] K. Severing and K. Saalwachter, Biaxial nematic phase in a thermotropic liquid-crystalline side-chain polymer, Phys. Rev. Lett., 92, 125501, 2004.

[33] S. Sircar and Q. Wang, Shear-induced mesostructures in biaxial liquid crystals, Phys. Rev. E,78, 061702, 2008.

[34] S. Sircar and Q. Wang, Submitted to J. Rheol., 061702, 2008.

[35] S. Sircar, Dynamics and Rheology of Biaxial Liquid Crystals, Ph. D. Thesis, University of South Carolina, 2009

[36] A.M. Sonnet, E.G. Virga and G.E. Durand, Dielectric shape dispersion and biaxial transitions in nematic liquid crystals, Phys. Rev. E, 67, 061701, 2003.

[37] J.P. Straley, Ordered phases of a liquid of biaxial particles, Phys. Rev. A, 10(5), 1881, 1974.

[38] P.I.C. Teixeira, A.J. Masters and B.M. Mulders, Biaxial nematic order in the hardboomerang fluid, Mol. Cryst. Liq. Cryst. Sci. Technol., 323, 167-189, 1998.

[39] B. Tjipto-Margo, The Onsager theory of the isotropic nematic liquid crystal transition: biaxial particles in uniaxial phases, J. Chem. Phys., 94, 4546, 1991.

[40] Q. Wang, A hydrodynamic theory for solutions of nonhomogeneous nematic liquid crystalline polymers of different configurations, J. Chem. Phys., 116, 9120, 2002.

[41] Q. Wang, S. Sircar and H. Zhou, Steady solutions of the Smoluchowski equation for nematic polymers under imposed fields, Commun. Math. Sci., 3(4), 605-620, 2005.

[42] L.J. Yu and A Saupe, Observation of a biaxial nematic phase in potassium laurate-1decanol-water mixtures, Phys. Rev. Lett., 45, 1000, 1980. 